\title{
Voting in collective stopping games
}

Citation for published version (APA):

Herings, P. J. J., \& Predtetchinski, A. (2013). Voting in collective stopping games. Maastricht University, Graduate School of Business and Economics. GSBE Research Memoranda No. 014 https://doi.org/10.26481/umagsb.2013014

Document status and date:

Published: 01/01/2013

DOI:

10.26481/umagsb.2013014

Document Version:

Publisher's PDF, also known as Version of record

\section{Please check the document version of this publication:}

- A submitted manuscript is the version of the article upon submission and before peer-review. There can be important differences between the submitted version and the official published version of record.

People interested in the research are advised to contact the author for the final version of the publication, or visit the DOI to the publisher's website.

- The final author version and the galley proof are versions of the publication after peer review.

- The final published version features the final layout of the paper including the volume, issue and page numbers.

Link to publication

\footnotetext{
General rights rights.

- You may freely distribute the URL identifying the publication in the public portal. please follow below link for the End User Agreement:

www.umlib.nl/taverne-license

Take down policy

If you believe that this document breaches copyright please contact us at:

repository@maastrichtuniversity.nl

providing details and we will investigate your claim.
}

Copyright and moral rights for the publications made accessible in the public portal are retained by the authors and/or other copyright owners and it is a condition of accessing publications that users recognise and abide by the legal requirements associated with these

- Users may download and print one copy of any publication from the public portal for the purpose of private study or research.

- You may not further distribute the material or use it for any profit-making activity or commercial gain

If the publication is distributed under the terms of Article $25 \mathrm{fa}$ of the Dutch Copyright Act, indicated by the "Taverne" license above, 


\section{Maastricht University}

P. J ean-J acques Herings,

Arkadi Predtetchinski

Voting in Collective Stopping Games

RM/13/014

\section{GSBE}

Maastricht University School of Business and Economics

Graduate School of Business and Economics

P.O Box 616

NL-6200 MD Maastricht

The Netherlands 


\title{
Voting in Collective Stopping Games
}

\author{
P. Jean-Jacques Herings and Arkadi Predtetchinski*
}

March 27, 2013

\begin{abstract}
At each moment in time, some alternative from a finite set is selected by a dynamic process. Players observe the alternative selected and sequentially cast a yes or a no vote. If the set of players casting a yes-vote is decisive for the alternative in question, the alternative is accepted and the game ends. Otherwise the next period begins. We refer to this class of problems as collective stopping problems. Collective choice games, quitting games, and coalition formation games are particular examples that fit nicely into this more general framework.

When the core of this game is non-empty, a stationary equilibrium in pure strategies is shown to exist. But in general, even mixed stationary equilibria may not exist in collective stopping games. We consider strategies that are pure and actionindependent, and allow for a limited degree of history dependence. Under such individual behavior, aggregate behavior can be conveniently summarized by a collective strategy. We consider collective strategies that are simple and induced by two-step game-plans and provide a constructive proof that this collection always contains a subgame perfect equilibrium. The existence of such an equilibrium is shown to imply the existence of a sequential equilibrium in an extended model with incomplete information. Collective equilibria are shown to be robust to perturbations in the dynamic process and in utilities. We apply our approach to the case with three alternatives exhibiting a Condorcet cycle and to the Baron-Ferejohn model of redistributive politics.
\end{abstract}

JEL Classification COde: C62, C72, C73, C78.

Keywords: Voting, Collective choice, Coalition formation, Subgame perfect equilibrium, Stationary equilibrium.

*We would like to thank Paulo Barelli, Francis Bloch, John Duggan, Jànos Flesch, Jean Guillaume Forand, Julio González Díaz, Harold Houba, Tasos Kalandrakis, Hans Peters, Philipp Strack, and William Thomson for very their helpful comments on the paper. 


\section{Introduction}

We study a class of games with the following features. At each moment in time, an alternative is selected by means of a dynamic process, and the chosen alternative is put to a vote. If the set of players who votes in favor of the alternative is decisive for the alternative in question, the alternative is implemented, each player receives a payoff as determined by the alternative, and the game ends. Otherwise, the game moves to the next period with the dynamic process selecting a new alternative, and the whole process repeats itself until some alternative is selected. This set-up is sufficiently general to encompass classical problems in the literatures on collective choice, quitting, and coalition formation as special cases.

Indeed, many applications in collective choice involve the sequential evaluation of alternatives by a committee. When a given alternative is rejected, the committee members will consider a new alternative in a subsequent time period. Moreover, in many cases, none of the committee members has full control over the contents of the new proposal. Compte and Jehiel (2010a) study such problems and mention recruitment decisions in which candidates are examined one by one, business decisions where financing capacity for projects is scarce, and family decisions concerning housing as particular examples.

The decision the committee has to make is whether to accept the current proposal and stop searching, or to reject the current proposal and wait for a better alternative to arrive. Penn (2009) argues that, even though in real-life political processes the proposals might be chosen strategically, it is a useful simplifying assumption to consider them as exogenously generated as the outcome of some random process. The rules of the agenda-setting might be extremely complex, and modeling such an agenda-setting process faithfully might be unnecessary and even undesirable. Roberts (2007), Penn (2009), and Compte and Jehiel (2010a) all assume that the new alternative is drawn from a fixed probability distribution. Nevertheless, in many cases it is more realistic to assume that the probability by which a particular new alternative is selected may depend on the characteristics of the current alternative. We allow for this much richer class of selection dynamics in this paper.

In many collective choice problems, it is natural to assume that the decision-making body and its approval rules are fixed. In other problems, the decision-making body or the approval rule depend on the proposal in question. For instance, in quitting games as studied in Solan (2005) and Mashiah-Yaakovi (2009), nature chooses a player and the chosen player decides whether the game should continue or stop. Such games are a variation on wars of attrition models, first analyzed by Maynard-Smith (1974), with economic applications like patent races, oligopoly exit games, and and all-pay auctions. 
In economic applications like oligopoly exit games, stopping decisions are usually taken by management teams that consist of several decision makers and where decision making takes place by majority or unanimity rule. This motivates the study of stopping problems where at each stage there is a collection of coalitions that can decide to stop the process and where the collection of decisive coalitions may depend on the alternative under consideration. The class of games that we study is therefore also sufficiently general to admit an interpretation of coalition formation. Under this interpretation, in each time period nature selects a coalition and an allocation of payoffs, which is implemented if the coalition members all approve. Contrary to standard non-cooperative models of coalition formation as in Bloch (1996), Okada (1996), Ray and Vohra (1999), or Compte and Jehiel (2010b), nature does not first select a player, who next proposes a coalition, but directly selects the coalition itself.

The core consists of those alternatives for which no alternative and associated decisive coalition exists that gives each decisive coalition member a strictly higher utility. We find that each core element, if it exists, naturally induces a subgame perfect equilibrium in pure stationary strategies. When the core is empty, however, subgame perfect equilibrium need not even exist in mixed stationary strategies, where the intuition for non-existence is closely related to the logic of the Condorcet paradox. In the presence of discounting or a positive probability of breakdown, stationary equilibria do exist, but might require the use of mixing. Our approach is to insist on pure strategies but allow for history-dependence. We cover both discounted and non-discounted payoffs as special cases.

Duggan's (2011) extremely rich coalitional bargaining framework encompasses many classical bargaining and coalition formation models as special cases and obtains equilibrium existence in mixed stationary strategies. In this paper we focus on equilibria in pure strategies, and relax the stationarity assumption. While Duggan studies the case with discounted payoffs, our results and techniques apply equally to discounted and non-discounted payoffs.

In the context of collective choice problems, history-dependent strategies have been considered by Bernheim and Slavov (2009) and Vartiainen (2011). While these papers use methods that do not belong to the domain of non-cooperative game theory, the authors point out the similarity of their solution concepts to a strategy in a dynamic noncooperative game. In both contributions the choice of the policy in a given period of time can be conditioned on the entire history of policies chosen up to that period. The model in Bernheim and Slavov (2009) describes situations where the policy is being reconsidered every time period. Likewise in Vartiainen (2011), who builds on the model by Konishi and Ray (2003), a current policy can be altered by an active coalition at any point in time. In 
contrast to these contributions, our approach is entirely non-cooperative.

For the same reasons Maskin and Tirole (2001) provide for the study of stationary strategies, i.e. reducing the multiplicity of equilibria in dynamic games, reducing the number of parameters to be estimated in econometric models, and amenability to simulation techniques, we are interested in the question whether there are relatively "simple" subgame perfect equilibria. Ideally we would like to know that some relatively small set of wellbehaved strategies always contains at least one subgame perfect equilibrium. Notwithstanding the fact that the set of behavior strategies in the games we consider is vastly infinite, we will provide a procedure that determines a subgame perfect equilibrium in a finite number of steps.

We start our analysis by restricting attention to pure action-independent strategies. Action-independence says that the vote of a player is not conditioned on the votes previously cast, whether in the same round of voting or in the past. Thus in order to play an action-independent strategy, a player need not remember how each individual player has voted so far, but only what alternatives have been voted on, and in fact turned down, so far. The condition of action-independence guarantees a degree of robustness of our result with respect to the precise specification of the voting stage of the game. In the basic model described above, the voting order is exogenously given and is fixed throughout the game, and players observe all previously taken actions. Under action-independence, however, each of these assumptions can be relaxed. Our existence result extends without any difficulty to a more general model where the voting order is history-dependent and/or probabilistic, and information on previously cast votes might be incomplete.

Under action-independence, the play of the game can be conveniently summarized by a so-called collective strategy. A collective strategy describes whether, after each history of alternatives generated by nature, the current proposal must be accepted or rejected. In particular, a collective strategy tells us how to continue the play of the game following a deviation, i.e. a rejection of an alternative that, according to the collective strategy, should have been accepted. We refer to such a deviation as an "unlawful" rejection. We show how to construct strategies for the players from a given collective strategy and how the concept of subgame perfect equilibrium can be reformulated in terms of collective strategies, leading to the concept of collective equilibrium.

A collective strategy is said to be simple if an unlawful rejection of a given alternative at any point in the game triggers the same continuation play. A simple collective strategy can thus be described by a relatively small amount of data: namely, the main game-plan according to which the game will be played until the first unlawful rejection occurs, and for every alternative a continuation game-plan that will be played following an unlawful 
rejection of the given alternative.

A particular example of a simple collective strategy is a stationary collective strategy. A stationary collective strategy consists of some target set of alternatives that are deemed acceptable. As soon as nature generates an alternative from the target set, it is accepted. More general than a stationary collective strategy is a collective strategy that is induced by stationary game-plans, since each unlawfully rejected alternative may lead to a particular set of alternatives that is targeted next. However, we show that a collective equilibrium induced by stationary game-plans may not exist.

A two-step game-plan is only slightly more complicated than a stationary game-plan. Instead of having one target set of alternatives, the players have two sets, a set of alternatives $X^{1}$ and a larger set of alternatives $X^{2}$. In a two-step game-plan, players wait $m$ periods for nature to select an alternative from $X^{1}$. If no such alternative is chosen from $X^{1}$ in the first $m$ rounds then, the players wait for an alternative from the set $X^{2}$ to be chosen. When the threshold $m$ is equal to zero, a two-step game-plan is stationary.

EXAMPLE 1.1: To motivate these definitions consider a three-player quitting game as defined in Solan (2005). There are three alternatives called $x_{1}, x_{2}$, and $x_{3}$, chosen by nature with equal probability. If nature chooses $x_{i}$, Player $i$ decides whether to stop the game or to continue. The payoffs are given in Table 1. For example, if nature chooses alternative $x_{1}$ and Player 1 decides to stop, Player 1 gets a payoff of 1, Player 2 a payoff of 7 , and Player 3 a payoff of 0 . If the game never stops, all payoffs are zero.

\begin{tabular}{|c|ccc|}
\hline & $x_{1}$ & $x_{2}$ & $x_{3}$ \\
\hline 1 & 1 & 0 & 3 \\
2 & 7 & 4 & 0 \\
3 & 0 & 7 & 4 \\
\hline
\end{tabular}

Table 1: A three-player quitting game.

The core of the game is empty as the preferences of the players exhibit a cycle: Player 1 prefers Player 3 to stop the game, Player 3 prefers Player 2 to stop it, and Player 2 prefers Player 1 to do so. We will show in Section 4 that the game has no subgame perfect stationary equilibria, whether in pure or mixed strategies, and in Section 8 that the game has no collective equilibrium induced by stationary game-plans.

Now consider two game-plans, called $g$ and $h: g$ is the game-plan that says "always stop" and $h$ is the game-plan that says "in period 0 , only stop if $x_{2}$ is chosen, and in all 
subsequent periods only stop if $x_{2}$ or $x_{3}$ is chosen." The game-plan $g$ is stationary while the game-plan $h$ is two-step with a threshold of 1.

We recursively define the simple collective strategy $f$ as follows. Start by following the game-plan $g$. Keep following a game-plan until the first unlawful rejection occurs. After an unlawful rejection of $x_{1}$, erase the memory of play and follow game-plan $h$ from scratch. After an unlawful rejection of $x_{2}$ or $x_{3}$, erase the memory of play and follow game-plan $g$ from scratch.

The collective strategy $f$ thus constructed is said to be induced by two-step gameplans, with the game-plan $g$ serving as both the main-game plan of $f$ and the punishment game-plan following an unlawful rejection of $x_{2}$ and $x_{3}$, and $h$ serving as a punishment game-plan following an unlawful rejection of $x_{1}$. As we show in Section 8, the collective strategy $f$ corresponds to a subgame perfect equilibrium in pure strategies.

Our main result claims that there always is a collective equilibrium induced by two-step game-plans. Moreover, we specify an iterative procedure that terminates in a finite number of steps with the appropriate two-step game-plans. More precisely, the procedure yields a set of acceptable alternatives and a set of viable game-plans. Acceptable alternatives are precisely those alternatives that can be accepted in some collective equilibrium induced by two-step game-plans, and viable game-plans are precisely those two-step game-plans that can be used to induce such equilibria.

We also show how to specify an a priori upper bound on the threshold of two-step game-plans, and how to construct a collective equilibrium that is induced by two-step game-plans satisfying this upper bound. The importance of this refinement is that the set of two-step game-plans satisfying this bound, as well as the strategies they induce, is finite. We demonstrate that, unlike stationary equilibrium, our equilibrium concept is generically robust to small perturbations in the dynamic process and in utilities.

We examine the class of games with three alternatives and an arbitrary number of players that exhibits the Condorcet paradox: a decisive coalition of players prefers the first alternative to the second, another decisive coalition of players the second alternative to the third, and another decisive coalition prefers the third alternative to the first. The example discussed before is a specific illustration. We show that collective equilibria have the immediate acceptance property. The first alternative that is generated by nature is accepted.

We also apply collective equilibrium to the Baron-Ferejohn model of redistributive politics. In that model a stationary equilibrium does exist. We prove that the payoffs sustained by collective equilibria induced by two-step game-plans converge to the payoffs of 
the stationary equilibrium when the units in which redistribution takes place are sufficiently small.

The rest of the paper is organized as follows. Section 2 introduces the basic model and Section 3 establishes the one-shot deviation principle. Section 4 discusses stationary strategies and the relation between equilibria in stationary strategies and the core. We also discuss examples that admit no subgame perfect equilibria in stationary strategies and examples where all stationary equilibria are in mixed strategies. Section 5 is devoted to pure action-independent strategies, Sections 6 to collective strategies, Section 7 to simple collective strategies, and Section 8 to two-step game-plans. Section 9 provides the main result, a constructive proof of existence of a collective equilibrium induced by two-step game-plans. Section 10 applies our solution concept to a class of examples exhibiting the Condorcet paradox and Section 11 presents an application to the Baron-Ferejohn model of redistributive politics. Section 12 demonstrates the robustness of our solution concept, and Section 13 extends the results to an incomplete information setting. Finally, Section 14 concludes.

\section{The model}

We consider a dynamic game $\Gamma=\left(N, X, \mu^{0}, \mu, \mathcal{C}, u\right)$. The set of players is $N$, a set with cardinality $n$. In each period $t=0,1, \ldots$ nature draws an alternative $x$ from a non-empty, finite set $X$. In period 0 alternatives are selected according to the probability distribution $\mu^{0}$ on $X$. In later periods, the selection of alternatives is governed by the Markov process $\mu$, where $\mu(y \mid x)$ denotes the probability that the current alternative is $y$ conditional on previous period's alternative being $x$. To keep notation and proofs as simple as possible, we assume that $\mu$ is irreducible, i.e. given any current alternative $x$ there is positive probability to reach any other alternative $y$ at some point in the future.

After the selection of an alternative, all players vote sequentially, each player casting a "y" or an "n" vote. For the sake of expositional simplicity, we assume that the order of voting $\prec$ is independent of the history of play and that each player observes the entire history of play preceding his own move, assumptions that can easily be avoided as we demonstrate in an extended model in Section 13.

The correspondence $\mathcal{C}: X \rightarrow 2^{N}$ associates to each alternative $x$ a collection of decisive coalitions $\mathcal{C}(x)$, a collection of subsets of $N$. Alternative $x$ is accepted if and only if the set of players who vote " $\mathrm{y}$ " on $x$ is a member of $\mathcal{C}(x)$. After the acceptance of an alternative, the game ends. Otherwise, the game proceeds to the next time period. The collection $\mathcal{C}(x)$ 
is assumed to be non-empty and monotonic. If $C \in \mathcal{C}(x)$ and $D \subset N$ is a superset of $C$, then $D \in \mathcal{C}(x)$. In case $\emptyset \in \mathcal{C}(x)$, alternative $x$ corresponds to a breakdown alternative. The monotonicity assumption on $\mathcal{C}(x)$ implies that, irrespective of the voting behavior of the players, the game ends after a breakdown alternative is selected. We allow for the existence of multiple breakdown alternatives.

Player $i \in N$ has a utility function $u_{i}: X \rightarrow \mathbb{R}_{+}$, where $u_{i}(x)$ is the utility player $i$ derives from the implementation of alternative $x$. In case of perpetual disagreement, every player's utility is zero. The profile of utility functions $\left(u_{i}\right)_{i \in N}$ is denoted by $u$. Although players are assumed not to discount utilities, the equivalent model with a positive probability $1-\delta$ of breakdown in every period is a special case of our model. It suffices to specify that one of the alternatives $x$ in $X$ is a breakdown alternative which is selected with probability $1-\delta$ in every period and leads to utility $u(x)=0$.

Let $A=\{\mathrm{y}, \mathrm{n}\}^{N}$ denote the set of players' joint actions in a voting stage of the game. The subset $A^{*}(x)$ of $A$ defined by

$$
A^{*}(x)=\left\{a \in A \mid\left\{i \in N \mid a^{i}=\mathrm{y}\right\} \in \mathcal{C}(x)\right\}
$$

is the set of joint actions which leads to the acceptance of alternative $x$.

Let $H_{i}$ be the set of all histories where player $i$ makes a decision. We define $H_{i}$ as the set of all sequences $\left(s_{0}, a_{0}, \ldots, s_{t-1}, a_{t-1}, s_{t}, a_{t}^{\prec i}\right)$ where $s_{0}, \ldots, s_{t}$ are alternatives in $X$, $a_{0}, \ldots, a_{t-1}$ are the actions by the players in the voting stages $0, \ldots, t-1$, and $a_{t}^{\prec i}$ is an element of the set $\{\mathrm{y}, \mathrm{n}\}^{\prec i}$, where $\prec i=\{j \in N \mid j \prec i\}$ is the set of players who vote before player $i$. Notice that only sequences $\left(s_{0}, a_{0}, \ldots, s_{t-1}, a_{t-1}, s_{t}, a_{t}^{\prec i}\right)$ such that $\mu^{0}\left(s_{0}\right)>0, a_{k} \notin A^{*}\left(s_{k}\right)$ for every $k \in\{0, \ldots, t-1\}$, and $\mu\left(s_{k+1} \mid s_{k}\right)>0$ for every $k \in\{0, \ldots, t-1\}$ can occur with positive probability. A behavioral strategy for player $i$ is a function $\sigma_{i}: H_{i} \rightarrow[0,1]$ where $\sigma_{i}(h)$ is the probability for player $i$ to play "y" at history $h \in H_{i}$.

Three important special cases of the model are collective choice games, quitting games, and coalition formation games.

Collective choice games are obtained as follows. Suppose that for every alternative $x$, $\mathcal{C}(x)$ consists of all coalitions with at least $q$ players. This represents a quota voting rule with $q$ being the size of the majority required for the approval of an alternative. Under this specification of decisive coalitions and $\mu$ being such that proposals are drawn from a fixed probability distribution in each period, our model is the discrete analogue of the model of Compte and Jehiel (2010a). It is also closely related to the model of Banks and Duggan (2000) once proposals are generated by an exogenous dynamic process rather than chosen endogenously by a recognized player. 
In the simple example of a quitting game with perfect information as defined in Solan and Vieille (2001), we have $X=\left\{x_{1}, \ldots, x_{n}\right\}, \mu\left(x_{i}\right)=1 / n$ for every $i \in N$, and $\mathcal{C}\left(x_{i}\right)=$ $\{C \subset N \mid i \in C\}$. In this case an alternative $x_{i}$ is accepted if and only if player $i$ votes in favor of acceptance.

Bloch and Diamantoudi (2011) consider coalition formation in hedonic games. The formation of a coalition $C \in 2^{N} \backslash\{\emptyset\}$ leads to utilities $u_{i}(C)$ for the members of coalition $C$ and to utility zero for players in $N \backslash C$. The variation on the game of Bloch and Diamantoudi (2011) where the game ends as soon as the first coalition forms and where nature chooses the coalition rather than the proposer is a special case of our set-up. It is obtained by choosing the non-empty coalitions as the alternatives and the collection of decisive coalitions for alternative $C$ given by $\mathcal{C}(C)=\{D \subset N \mid D \supset C\}$.

More generally, since our set of decisive coalitions is allowed to depend on the alternative, we can also interpret our set-up as a coalition formation game. Let $X(C)$ denote the subset of alternatives in $X$ for which $C \in \mathcal{C}(x)$. Then $u(X(C))$ corresponds to the payoff set of coalition $C$. Compared to standard models of non-transferable utility games, our approach also specifies the payoffs to non-coalition members, so externalities are allowed for. Our monotonicity assumption on $\mathcal{C}$ leads to a monotonicity assumption on the sets of payoffs: if $\bar{u} \in u(X(C))$ and $C \subset D$, then $\bar{u} \in u(X(D))$.

The game $\Gamma$ belongs to the class of stochastic games with perfect information and recursive payoffs. These are stochastic games where each state is controlled by one player, the payoffs in the transient states are all zero, and the payoffs in the absorbing states are non-negative. The main result in Flesch, Kuipers, Schoenmakers, and Vrieze (2010) states that $\Gamma$ has a subgame perfect $\epsilon$-equilibrium in pure strategies for every $\epsilon>0$. As is explained in detail in the following sections, we exploit the special features of the game $\Gamma$ to obtain significantly stronger results.

\section{The one-shot deviation principle}

The one-shot deviation principle claims that a joint strategy $\sigma=\left(\sigma_{i}\right)_{i \in N}$ is a subgame perfect equilibrium if and only if no player $i$ has a strategy $\sigma_{i}^{\prime}$ such that $\sigma_{i}^{\prime}$ agrees with $\sigma_{i}$ after all histories in $H_{i}$ except some history $h$, and conditional on history $h, \sigma_{i}^{\prime}$ yields player $i$ a higher payoff against $\sigma_{-i}$ than $\sigma_{i}$. A strategy $\sigma_{i}^{\prime}$ as above is said to be a profitable one-shot deviation from $\sigma$. It is well-known that the one-shot deviation principle holds in any dynamic game where the payoff function is continuous at infinity, see for instance Fudenberg and Tirole (1991), Theorem 4.2. Unfortunately, the payoff function in our game 
$\Gamma$ is not continuous at infinity. Nevertheless, as demonstrated below, our game does satisfy the one-shot deviation principle.

Let a joint strategy $\sigma$ and a history $h \in H_{i}$ be given. We let $\pi(t, x \mid \sigma, h)$ denote the probability that the play of the game will terminate in period $t$ with the acceptance of alternative $x$, conditional on the fact that history $h$ has taken place. The expected payoff of player $i$ conditional on history $h$ is given by

$$
v_{i}(\sigma \mid h)=\sum_{x \in X} u_{i}(x) \sum_{t=0}^{\infty} \pi(t, x \mid \sigma, h) .
$$

A joint strategy $\sigma$ is a subgame perfect equilibrium of the game $\Gamma$ if for every player $i$, each history $h \in H_{i}$, and each strategy $\sigma_{i}^{\prime}$ it holds that

$$
v_{i}\left(\sigma_{i}^{\prime}, \sigma_{-i} \mid h\right) \leq v_{i}\left(\sigma_{i}, \sigma_{-i} \mid h\right)
$$

TheOREm 3.1: (The one-shot deviation principle) A joint strategy $\sigma$ is a subgame perfect equilibrium of $\Gamma$ if and only if for every player $i$, each $h^{\prime} \in H_{i}$, and each strategy $\sigma_{i}^{\prime}$ such that $\sigma_{i}^{\prime}(h)=\sigma_{i}(h)$ for every $h \in H_{i} \backslash\left\{h^{\prime}\right\}$, it holds that

$$
v_{i}\left(\sigma_{i}^{\prime}, \sigma_{-i} \mid h^{\prime}\right) \leq v_{i}\left(\sigma_{i}, \sigma_{-i} \mid h^{\prime}\right) \text {. }
$$

The "only if" part of the theorem is trivial. The lemma below is a crucial step towards the proof of the "if" part. The result claims that if a player $i$ can improve upon his payoff obtained under $\sigma$, he can do so by deviating from $\sigma$ at finitely many histories in $H_{i}$ only.

Let $\sigma_{i}$ and $\sigma_{i}^{\prime}$ be strategies for player $i$. For each $k \in \mathbb{N}$, we define the strategy $\sigma_{i}^{k}$ for player $i$ by the following rule: The strategy $\sigma_{i}^{k}$ agrees with $\sigma_{i}^{\prime}$ for histories $h \in H_{i}$ in periods $0, \ldots, k$, and agrees with $\sigma_{i}$ for histories $h \in H_{i}$ in periods $k+1, k+2, \ldots$. Formally, for a history $h=\left(s_{0}, a_{0}, \ldots, s_{t-1}, a_{t-1}, s_{t}, a_{t}^{\prec i}\right)$ in $H_{i}$, set $\sigma_{i}^{k}(h)=\sigma_{i}^{\prime}(h)$ if $t \leq k$ and set $\sigma_{i}^{k}(h)=\sigma_{i}(h)$ otherwise.

LEMMA 3.2: Let $\sigma_{i}$ and $\sigma_{i}^{\prime}$ be strategies for player $i$ and let $\sigma_{-i}$ be a tuple of strategies for the other players. Suppose that for some $h \in H_{i}$ we have that

$$
v_{i}\left(\sigma_{i}^{\prime}, \sigma_{-i} \mid h\right)>v_{i}\left(\sigma_{i}, \sigma_{-i} \mid h\right)
$$

Then there exists a $k \in \mathbb{N}$ such that

$$
v_{i}\left(\sigma_{i}^{k}, \sigma_{-i} \mid h\right)>v_{i}\left(\sigma_{i}, \sigma_{-i} \mid h\right)
$$


Proof: We write $\sigma=\left(\sigma_{i}, \sigma_{-i}\right), \sigma^{\prime}=\left(\sigma_{i}^{\prime}, \sigma_{-i}\right)$, and $\sigma^{k}=\left(\sigma_{i}^{k}, \sigma_{-i}\right)$. Choose $\epsilon>0$ such that $v_{i}\left(\sigma^{\prime} \mid h\right)>v_{i}(\sigma \mid h)+\epsilon$. Choose $c>0$ such that for every $i \in N, x \in X$, it holds that $u_{i}(x) \leq c$. Let $t_{h}$ denote the time period of history $h$. The sum

$$
\sum_{t=t_{h}}^{\infty} \sum_{x \in X} \pi\left(t, x \mid \sigma^{\prime}, h\right)
$$

is the probability that the game eventually ends with the acceptance of some alternative, and hence is bounded from above by 1 . Therefore, there is a time period $k \geq t_{h}$ such that

$$
\sum_{t=k+1}^{\infty} \sum_{x \in X} \pi\left(t, x \mid \sigma^{\prime}, h\right) \leq \frac{\epsilon}{c}
$$

and it holds that

$$
v_{i}\left(\sigma^{\prime} \mid h\right)-\sum_{t=t_{h}}^{k} \sum_{x \in X} u_{i}(x) \pi\left(t, x \mid \sigma^{\prime}, h\right)=\sum_{t=k+1}^{\infty} \sum_{x \in X} u_{i}(x) \pi\left(t, x \mid \sigma^{\prime}, h\right) \leq \epsilon .
$$

Since the joint strategies $\sigma^{k}$ and $\sigma^{\prime}$ agree on all histories up to and including period $k$, we have $\pi\left(t, x \mid \sigma^{k}, h\right)=\pi\left(t, x \mid \sigma^{\prime}, h\right)$ whenever $t_{h} \leq t \leq k$. Hence

$$
\begin{aligned}
v_{i}\left(\sigma^{k} \mid h\right) & \geq \sum_{t=t_{h}}^{k} \sum_{x \in X} u_{i}(x) \pi\left(t, x \mid \sigma^{k}, h\right) \\
& =\sum_{t=t_{h}}^{k} \sum_{x \in X} u_{i}(x) \pi\left(t, x \mid \sigma^{\prime}, h\right) \geq v_{i}\left(\sigma^{\prime} \mid h\right)-\epsilon>v_{i}(\sigma \mid h),
\end{aligned}
$$

where the first inequality follows since $u(x) \geq 0$ for every $x \in X$.

The one-shot deviation principle can now be derived using a standard technique.

Proof of Theorem 3.1: Suppose there is a player $i$, a history $h \in H_{i}$, and a strategy $\sigma_{i}^{\prime}$ such that

$$
v_{i}\left(\sigma_{i}^{\prime}, \sigma_{-i} \mid h\right)>v_{i}\left(\sigma_{i}, \sigma_{-i} \mid h\right) .
$$

By Lemma 3.2 there exists $k$ such that $v_{i}\left(\sigma_{i}^{k}, \sigma_{-i} \mid h\right)>v_{i}\left(\sigma_{i}, \sigma_{-i} \mid h\right)$. Either there is a history $h^{\prime}$ in period $k$ where player $i$ has a profitable one-shot deviation from $\sigma_{i}$, or

$$
v_{i}\left(\sigma_{i}^{k-1}, \sigma_{-i} \mid h\right)>v_{i}\left(\sigma_{i}, \sigma_{-i} \mid h\right)
$$

This process terminates in finitely many steps with a history where player $i$ has a profitable one-shot deviation. $\square$ 


\section{Stationary strategies}

It has been shown in Fink (1964), Takahashi (1964), and Sobel (1971) that a stochastic game with discounting admits a subgame perfect equilibrium in stationary strategies. When at least one of the alternatives in our model is a breakdown alternative, the techniques of the stochastic game literature with discounting can be used to show the existence of a subgame perfect equilibrium in stationary strategies. However, it is well-known that even in the presence of discounting, subgame perfect equilibria in pure stationary strategies may not exist.

In the absence of discounting, even when allowing for mixed strategies, non-existence of a Nash equilibrium has been noted by Blackwell and Ferguson (1968) and obviously implies the non-existence of a subgame perfect equilibrium in stationary strategies. This result has spurred an extensive literature on the existence of weaker notions of Nash equilibrium in special classes of stochastic games with the average reward criterion. An example is the class of recursive games with positive payoffs as introduced in Flesch et al. (2010), a class for which they show the existence of a subgame perfect $\epsilon$-equilibrium in pure strategies. Since our model belongs to the class of recursive games with positive payoffs, this result immediately applies.

We study next whether $\Gamma$ has subgame perfect equilibria in stationary strategies. We say that a strategy is stationary if the probability to vote in favor of a given alternative $x$ depends only on $x$ and is otherwise independent of the history of play. ${ }^{1}$

Definition 4.1: A strategy $\sigma_{i}$ for player $i$ is stationary if for all histories $h=\left(s_{0}, a_{0}, \ldots\right.$, $\left.s_{t-1}, a_{t-1}, s_{t}, a_{t}^{\prec i}\right)$ and $h^{\prime}=\left(s_{0}^{\prime}, a_{0}^{\prime}, \ldots, s_{t^{\prime}-1}^{\prime}, a_{t^{\prime}-1}^{\prime}, s_{t^{\prime}}^{\prime}, a_{t^{\prime}}^{\prime<i}\right)$ in $H_{i}$ such that $s_{t}=s_{t^{\prime}}^{\prime}$ it holds that $\sigma_{i}(h)=\sigma_{i}\left(h^{\prime}\right)$.

Let $x$ and $y$ be alternatives in $X$. The alternative $x$ is said to strictly dominate $y$ if

$$
\left\{i \in N \mid u_{i}(x)>u_{i}(y)\right\} \in \mathcal{C}(x)
$$

Notice that under the maintained assumptions it is possible that $x$ strictly dominates $y$ while at the same time $y$ strictly dominates $x$. The set of alternatives that strictly dominate $y$ is denoted by $\operatorname{SD}(y)$. An alternative $x$ is said to have the core property if it is not strictly dominated by any other alternative. The core consists of all alternatives with the core

\footnotetext{
${ }^{1}$ Our notion of stationary is somewhat more stringent than the usual one in the literature. Following the approach in Maskin and Tirole (2001) would lead to a notion of stationarity where the voting decision of a player is allowed to depend on the votes cast previously in the current round of voting. Our negative results regarding the existence of stationary equilibria carry over to this weaker notion of stationarity.
} 
property. Our definition of the core equals the one as given for effectivity functions, see Definition 5.2.5 of Peleg and Peters (2010).

For non-transferable utility games, the core is defined in terms of utilities rather than alternatives, and more precisely as those utilities $\bar{u} \in u(X(N))$ for which there is no $C \subset N$ with $\hat{u} \in u(X(C))$ such that $\hat{u}_{i}>\bar{u}_{i}$ for all $i \in C$. The monotonicity property of $\mathcal{C}$ implies the consistency of our definition of the core with the one in the theory on non-transferable utility games.

It follows directly from the definition that a breakdown alternative strictly dominates any alternative including itself. Non-emptiness of the core therefore implies the absence of breakdown alternatives.

Let $\Gamma$ have a non-empty core, and let $\bar{x} \in X$ be an alternative with the core property. We define the following pure stationary strategy for player $i \in N$ :

$$
\sigma_{i}\left(s_{0}, a_{0}, \ldots, s_{t-1}, a_{t-1}, s_{t}, a_{t}^{\prec i}\right)= \begin{cases}\mathrm{y}, & \text { if } s_{t}=\bar{x} \text { or } u_{i}\left(s_{t}\right)>u_{i}(\bar{x}), \\ \mathrm{n}, & \text { otherwise. }\end{cases}
$$

Under the joint strategy $\sigma$, all players vote in favor of alternative $\bar{x}$, and since $\mathcal{C}(\bar{x})$ is non-empty and monotonic, $\bar{x}$ is accepted whenever drawn by nature.

TheOrem 4.2: Let $\Gamma$ have a non-empty core. The joint strategy $\sigma$ as defined in (4.1) is a subgame perfect equilibrium in pure stationary strategies.

Proof: Let $\bar{x} \in X$ be an alternative with the core property. We define the pure stationary strategy $\sigma_{i}$ for player $i \in N$ by (4.1). Under the joint strategy $\sigma$, it clearly holds that $\bar{x}$ is accepted whenever drawn by nature.

Suppose there is another alternative, say $x$, which is accepted when drawn by nature. By definition of $\sigma$ it holds that $\left\{i \in N \mid u_{i}(x)>u_{i}(\bar{x})\right\} \in \mathcal{C}(x)$, which means that $x$ strictly dominates $\bar{x}$, a contradiction. Consequently, $\bar{x}$ is the only alternative that will ever be accepted.

Since $\mu$ is irreducible and $\bar{x}$ is the only alternative that will ever be accepted, it holds that $v(\sigma)=u(\bar{x})$. Moreover, the expected utility to the players following the rejection of any alternative is given by $u(\bar{x})$.

We verify next that there are no profitable one-shot deviations from $\sigma$. Consider a history $h$ at which, according to $\sigma$, player $i$ has to vote in favor of an alternative $x$. By definition of $\sigma$ it holds that $u_{i}(x) \geq u_{i}(\bar{x})$. When the play resulting from $\sigma$ leads to the acceptance of $x$, we have that $v_{i}(\sigma \mid h)=u_{i}(x)$, whereas a one-shot deviation by player $i$ to a vote against either still results in the acceptance of $x$, or to a rejection and utility 
$u_{i}(\bar{x}) \leq u_{i}(x)$, so is not profitable. When playing according to $\sigma$ leads to the rejection of $x$, a one-shot deviation by player $i$ to a vote against will still lead to the rejection of $x$ by monotonicity of $\mathcal{C}(x)$, and is therefore not profitable. Consider a history $h$ at which, according to $\sigma$, player $i$ has to vote against an alternative $x$. By definition of $\sigma$ we have that $u_{i}(x) \leq u_{i}(\bar{x})$. By monotonicity of $\mathcal{C}(x)$, a one-shot deviation by player $i$ to a vote in favor will either not make a difference or change a rejection of $x$ into an acceptance and lead to utility $u_{i}(x) \leq u_{i}(\bar{x})$, so is not profitable.

By Theorem 3.1 we conclude that $\sigma$ is a subgame perfect equilibrium.

The following example of a collective choice game illustrates that even when the core is non-empty, there may be subgame perfect equilibria in pure stationary strategies leading to the acceptance of an alternative that does not belong to the core. In fact, even in the presence of an alternative that is unanimously preferred to all other alternatives, some of the other alternatives might be accepted.

EXAMPLE 4.3: There are 3 players and 4 alternatives with payoffs given by Table 2 on the left. The collection of decisive coalitions consists of all coalitions with two or more players, so is obtained by an application of simple majority rule. In every time period, all alternatives have an equal chance to be selected by nature. Notice that alternative $x_{4}$ is strictly preferred by all players to any other alternative. The alternative $x_{4}$ has the core property and Theorem 4.2 implies that there is a subgame perfect equilibrium in pure stationary strategies where an alternative is accepted by a majority if and only if it is alternative $x_{4}$. However, there is another subgame perfect equilibrium in pure stationary strategies given by Table 2 on the right. In this equilibrium all four alternatives are immediately accepted, resulting in an expected payoff of 15/4 for every player. Since $15 / 4<4$ and 4 is the minimum utility of a player who casts a yes vote, it is easy to verify that the one-shot deviation principle is satisfied, and the strategy is a subgame perfect equilibrium indeed.

\begin{tabular}{|c|cccc|}
\hline & $x_{1}$ & $x_{2}$ & $x_{3}$ & $x_{4}$ \\
\hline 1 & 5 & 4 & 0 & 6 \\
2 & 0 & 5 & 4 & 6 \\
3 & 4 & 0 & 5 & 6 \\
\hline
\end{tabular}

\begin{tabular}{|c|cccc|}
\hline & $x_{1}$ & $x_{2}$ & $x_{3}$ & $x_{4}$ \\
\hline 1 & $\mathrm{y}$ & $\mathrm{y}$ & $\mathrm{n}$ & $\mathrm{y}$ \\
2 & $\mathrm{n}$ & $\mathrm{y}$ & $\mathrm{y}$ & $\mathrm{y}$ \\
3 & $\mathrm{y}$ & $\mathrm{n}$ & $\mathrm{y}$ & $\mathrm{y}$ \\
\hline
\end{tabular}

Table 2: Payoffs and strategies in Example 4.3. 


\begin{tabular}{|c|ccc|}
\hline & $x_{1}$ & $x_{2}$ & $x_{3}$ \\
\hline 1 & 1 & 0 & 3 \\
2 & 7 & 4 & 0 \\
3 & 0 & 7 & 4 \\
\hline
\end{tabular}

Table 3: Payoffs in Example 4.4.

The following two examples illustrate that when $\Gamma$ has an empty core, there might not be a subgame perfect equilibrium in mixed stationary strategies. The first example corresponds to a quitting game, the second example is a collective choice game that exhibits the Condorcet paradox. The second example can also be reformulated as a coalition formation game. This example is closely related to an example on coalition formation where Bloch (1996) shows non-existence of a subgame perfect equilibrium in stationary strategies.

EXAMPLE 4.4: This is an example of a 3-player quitting game with perfect information as studied in Solan (2005). The set of players is $N=\{1,2,3\}$ and the set of alternatives is $X=\left\{x_{1}, x_{2}, x_{3}\right\}$. The utilities of these alternatives are specified in Table 3 . In every time period, every alternative is chosen by nature with probability $1 / 3$. Player $i$ is decisive for alternative $x_{i}$, that is $\mathcal{C}\left(x_{i}\right)=\{C \subset N \mid i \in C\}$. It holds that $x_{3}$ strictly dominates $x_{1}, x_{1}$ strictly dominates $x_{2}$, and $x_{2}$ strictly dominates $x_{3}$, so the core of the game is empty, and Theorem 4.2 cannot be applied.

In this example, a stationary strategy of player $i$ can be represented by a number $\alpha_{i} \in[0,1]$, being the probability for player $i$ to vote in favor of alternative $x_{i}$. In a stationary strategy, the vote of player $i$ on the other alternatives is inconsequential, and can therefore be ignored. For $i \in N$, it holds that $v_{i}(0,0,0)=0$ and

$$
v_{i}(\alpha)=\frac{1}{\alpha_{1}+\alpha_{2}+\alpha_{3}}\left(\alpha_{1} u_{i}\left(x_{1}\right)+\alpha_{2} u_{i}\left(x_{2}\right)+\alpha_{3} u_{i}\left(x_{3}\right)\right), \quad \alpha \neq(0,0,0) .
$$

By stationarity, $v(\alpha)=\left(v_{1}(\alpha), v_{2}(\alpha), v_{3}(\alpha)\right)$ is also the expected utility conditional on the rejection of any alternative. By Theorem 3.1 it holds that the joint stationary strategy $\alpha$ is subgame perfect if and only if for every player $i$

$$
\begin{array}{lll}
\alpha_{i}>0 & \text { implies } & u_{i}\left(x_{i}\right) \geq v_{i}(\alpha), \\
\alpha_{i}<1 & \text { implies } & u_{i}\left(x_{i}\right) \leq v_{i}(\alpha) .
\end{array}
$$

We claim that the game has no subgame perfect equilibrium in stationary strategies. For suppose $\alpha$ is such a strategy. We split the argument into four cases depending on the number $m$ of non-zero components of $\alpha$. 
Case $m=0$. We have that $\alpha=(0,0,0)$ and $v(\alpha)=(0,0,0)$. Since $u_{1}\left(x_{1}\right)=1>0=$ $v_{1}(\alpha)$, we must have $\alpha_{1}=1$, a contradiction.

Case $m=1$. Suppose first that $\alpha_{1}>0$. It follows that $v(\alpha)=u\left(x_{1}\right)$. But then $u_{3}\left(x_{3}\right)=4>0=v_{3}(\alpha)$ and so we must have $\alpha_{3}=1$, implying that $m \geq 2$. Similarly, if $\alpha_{2}>0$ a contradiction arises since $v(\alpha)=u\left(x_{2}\right)$ and $u_{1}\left(x_{1}\right)=1>0=v_{1}(\alpha)$, and if $\alpha_{3}>0$ a contradiction arises since $v(\alpha)=u\left(x_{3}\right)$ and $u_{2}\left(x_{2}\right)=4>0=v_{2}(\alpha)$.

Case $m=2$. Suppose first that $\alpha_{3}=0$. Then $v(\alpha)$ is a strictly convex combination of $u\left(x_{1}\right)$ and $u\left(x_{2}\right)$ and hence $v_{2}(\alpha)>u_{2}\left(x_{2}\right)$. Hence $\alpha_{2}=0$, implying that $m \leq 1$, a contradiction. Similarly, if $\alpha_{2}=0$ a contradiction arises since $v_{1}(\alpha)>u_{1}\left(x_{1}\right)$, and if $\alpha_{1}=0$ a contradiction arises since $v_{3}(\alpha)>u_{3}\left(x_{3}\right)$.

Case $m=3$. We have $\alpha_{1}, \alpha_{2}, \alpha_{3}>0$ and so

$$
u_{i}\left(x_{i}\right) \geq v_{i}(\alpha)=\frac{1}{\alpha_{1}+\alpha_{2}+\alpha_{3}}\left(\alpha_{1} u_{i}\left(x_{1}\right)+\alpha_{2} u_{i}\left(x_{2}\right)+\alpha_{3} u_{i}\left(x_{3}\right)\right), \quad i \in N .
$$

Rewriting leads to the inequalities

$$
\alpha_{3} / \alpha_{2} \leq 1 / 2, \alpha_{1} / \alpha_{3} \leq 4 / 3, \text { and } \alpha_{2} / \alpha_{1} \leq 4 / 3 \text {, }
$$

and therefore

$$
1=\frac{\alpha_{3}}{\alpha_{2}} \frac{\alpha_{1}}{\alpha_{3}} \frac{\alpha_{2}}{\alpha_{1}} \leq \frac{1}{2} \cdot \frac{4}{3} \cdot \frac{4}{3}=\frac{8}{9},
$$

a contradiction.

EXAMPLE 4.5: All the primitives are the same as in the preceding example, except for the collection of decisive coalitions. Suppose that the votes of two out of three players are needed for the acceptance of an alternative, so $\mathcal{C}(x)$ consists of all subsets of $N$ with at least two players. This is an example of a Condorcet paradox, where the majority induced preference relation is intransitive. In a pairwise comparison, alternative $x_{1}$ beats $x_{2}$, alternative $x_{2}$ beats $x_{3}$, and alternative $x_{3}$ beats $x_{1}$. Herings and Houba (2010) study this example under the alternative model where the proposer rather than the alternative is selected by nature.

We claim that the game admits no subgame perfect equilibrium in stationary strategies. A stationary strategy of player $i$ in this game specifies, for every alternative $x$, the probability for player $i$ to vote in favor of $x$.

Suppose the joint stationary strategy $\sigma$ is a subgame perfect equilibrium. It is rather straightforward, though tedious, to show that the cases where $\sigma$ leads to the rejection of all alternatives, or to the acceptance of at most one alternative with positive probability, are 


\begin{tabular}{|c|cccc|}
\hline & $x_{0}$ & $x_{1}$ & $x_{2}$ & $x_{3}$ \\
\hline 1 & 0 & 1 & 0 & 5 \\
2 & 0 & 5 & 1 & 0 \\
3 & 0 & 0 & 5 & 1 \\
\hline
\end{tabular}

Table 4: Payoffs in Example 4.6.

not compatible with equilibrium. Equilibrium utilities are therefore strictly in between the utility of the worst and the best alternative for every player. Next it is rather straightforward, though tedious as well, to show that each player votes in favor of his best alternative and against his worst alternative with probability one.

Hence, in essence, every player $i$ is decisive for alternative $x_{i}$ since there is one more player who votes in favor of $x_{i}$ and one more who votes against it. Thus the joint stationary strategy $\sigma$ induces a subgame perfect equilibrium of the game of Example 4.4, contradicting the conclusion that no stationary subgame perfect equilibria exist in that game. Consequently, no joint stationary strategy $\sigma$ can be a subgame perfect equilibrium in the game of Example 4.5.

Example 4.5 studies the case of majority voting in a three-player set-up with an empty core, or equivalently, the absence of a Condorcet winner. It has been shown in the literature that the occurrence of the Condorcet paradox is not an artifact. Work by Plott (1967), Rubinstein (1979), Schofield (1983), Cox (1984), and Le Breton (1987) shows that the core is generically empty in majority voting situations with three or more players in a set-up where alternatives are a compact, convex subset of some Euclidean space. Such voting situations can be approximated arbitrarily closely in our set-up with a finite set of alternatives.

The next example of a quitting game shows that in the presence of a breakdown alternative, subgame perfect equilibria in pure stationary strategies may fail to exist, and that subgame perfect equilibria in mixed stationary strategies, which do exist in this case, are Pareto inefficient.

EXAMPLE 4.6: In this example the set of players is $N=\{1,2,3\}$ and the set of alternatives is $X=\left\{x_{0}, x_{1}, x_{2}, x_{3}\right\}$. The utilities of these alternatives are specified in Table 4 . Every period a breakdown alternative $x_{0}$ is selected with a probability $1-\delta$ strictly in between 0 and 1. For the other alternatives it holds that player $i$ is decisive for alternative $x_{i}$, which is selected with probability $\delta / 3$ in every period. 
In this example, a stationary strategy of player $i$ can be represented by a number $\alpha_{i} \in[0,1]$, being the probability for player $i$ to vote in favor of alternative $x_{i}$. In a stationary strategy, the vote of player $i$ on other alternatives is inconsequential, and can therefore be ignored. For $i \in N$, it holds that

$$
v_{i}(\alpha)=\frac{1}{3-3 \delta+\delta \alpha_{1}+\delta \alpha_{2}+\delta \alpha_{3}}\left(\delta \alpha_{1} u_{i}\left(x_{1}\right)+\delta \alpha_{2} u_{i}\left(x_{2}\right)+\delta \alpha_{3} u_{i}\left(x_{3}\right)\right) .
$$

By stationarity, $v(\alpha)=\left(v_{1}(\alpha), v_{2}(\alpha), v_{3}(\alpha)\right)$ is also the expected utility conditional on the rejection of any alternative. By Theorem 3.1 it holds that the joint stationary strategy $\alpha$ is subgame perfect if and only if for every player $i \in N$,

$$
\begin{array}{lll}
\alpha_{i}>0 & \text { implies } & u_{i}\left(x_{i}\right) \geq v_{i}(\alpha), \\
\alpha_{i}<1 & \text { implies } & u_{i}\left(x_{i}\right) \leq v_{i}(\alpha) .
\end{array}
$$

It is a routine exercise to verify that there is a unique subgame perfect equilibrium in stationary strategies. If $\delta \leq 1 / 2$, then it is given by the pure strategy profile $\alpha_{1}=\alpha_{2}=$ $\alpha_{3}=1$ with expected payoffs $v(\alpha)=(2 \delta, 2 \delta, 2 \delta)$. If $\delta>1 / 2$, then it is given by the mixed strategy profile $\alpha_{1}=\alpha_{2}=\alpha_{3}=(1-\delta) / \delta$ with expected payoffs $v(\alpha)=(1,1,1)$, irrespective of $\delta$. A strategy profile that would lead to immediate acceptance of every alternative has payoffs $(2 \delta, 2 \delta, 2 \delta)$, which converges to $(2,2,2)$ as $\delta$ tends to 1 . The delay in the unique stationary subgame perfect equilibrium causes substantial inefficiencies. The expected delay before reaching an agreement is equal to $(2 \delta-1) /(2-2 \delta)$ periods, which tends to infinity as $\delta$ tends to 1 . In the limit, every alternative is rejected with probability 1.

\section{Action-independence}

The objective of this paper is to prove the existence of a subgame perfect equilibrium in the game $\Gamma$. Moreover, we would like to construct a subgame perfect equilibrium in pure strategies that exhibits a relatively small amount of history-dependence and that is computable in finitely many steps. To guarantee the existence of a subgame perfect equilibrium, some history-dependence is needed as is evidenced by Examples 4.4 and 4.5. Example 4.6 shows that even in the presence of breakdown alternatives, some historydependence is needed to guarantee the existence of a subgame perfect equilibrium in pure strategies.

We will consider strategies that are pure and action-independent. A strategy is said to be action-independent if it prescribes the same action after the same sequence of moves 
by nature. In other words, under action-independence a player is not allowed to condition his vote on the actions of the players, but only on the moves by nature.

Definition 5.1: A strategy $\sigma_{i}$ for player $i$ is action-independent if for all histories $h=$ $\left(s_{0}, a_{0}, \ldots, s_{t-1}, a_{t-1}, s_{t}, a_{t}^{\prec i}\right)$ and $\bar{h}=\left(\bar{s}_{0}, \bar{a}_{0}, \ldots, \bar{s}_{t-1}, \bar{a}_{t-1}, \bar{s}_{t}, \bar{a}_{t}^{\prec i}\right)$ in $H_{i}$ such that $s_{0}=$ $\bar{s}_{0}, \ldots, s_{t}=\bar{s}_{t}$, it holds that $\sigma_{i}(h)=\sigma_{i}(\bar{h})$.

The requirement of action-independence guarantees the robustness of our solution with respect to the exact set-up of the voting stage of the game. We presently assume that the players vote on each proposal sequentially, that the order of voting is fixed, and that the players observe all moves previously made. Action-independence implies that the order of voting is inessential. Accordingly, our existence result carries over without any difficulty to a game where the order of voting is random or history-dependent. Moreover, under actionindependence it is inessential whether the players are indeed able to observe previously cast ballots or not. Consequently, our existence result extends to a game where the players have various degrees of incomplete information about the voting behavior of other players. In particular, we encompass the situation where the players cast their votes simultaneously and the votes are not, partially, or completely disclosed at the end of the voting stage. Section 13 presents the details for such an extension to incomplete information settings.

\section{Collective strategies}

Under action-independence, the play of the game can be conveniently summarized by means of so-called collective strategies. A collective strategy is a complete contingent plan of actions which specifies whether a given alternative has to be accepted or rejected, following a given sequence of alternatives selected by nature.

Let $S$ be the set of all finite sequences of elements of $X$. A collective strategy is a function $f$ from $S$ to $\{0,1\}$, where 0 corresponds to a rejection and 1 to an acceptance. We define $f(s)$ for every element $s \in S$, so $f$ also specifies how the game is played for counterfactual situations where the game proceeds after the acceptance of an alternative. Let $F$ be the set of functions from $S$ to $\{0,1\}$.

Consider a joint strategy $\sigma$ where $\sigma_{i}$ is pure and action-independent for each player $i \in N$. The collective strategy $f_{\sigma}: S \rightarrow\{0,1\}$ induced by $\sigma$ is defined by

$$
f_{\sigma}\left(s_{0}, \ldots, s_{t}\right)= \begin{cases}1, & \text { if }\left\{i \in N \mid \sigma_{i}\left(s_{0}, \ldots, s_{t}\right)=\mathrm{y}\right\} \in \mathcal{C}\left(s_{t}\right) \\ 0, & \text { otherwise }\end{cases}
$$


Here we have used action-independence to treat $\sigma_{i}$ as a function with domain $S$ rather than $H_{i}$. Thus $f_{\sigma}(s)=1$ if and only if $s$ is accepted according to the joint strategy $\sigma$. Notice that $f_{\sigma}(s)=1$ if $s_{t}$ is a breakdown alternative, irrespective of the actual voting behavior of the players.

Given some $x \in X$, we let $\Gamma_{x}$ denote the game where the initial probability distribution $\mu^{0}$ over $X$ is defined by $\mu^{0}(y)=\mu(y \mid x), y \in X$. We let $V_{x}(f)$ denote the vector of expected payoffs in the game $\Gamma_{x}$, if play proceeds according to the collective strategy $f$. We shall often consider the case where the Markov process $\mu$ is stationary, that is $\mu(z \mid x)=\mu(z \mid y)$ for all $x, y$, and $z$. In this special case $V_{x}(f)$ is clearly the same for each $x \in X$, and we shall simply write $V(f)$ to denote this common value.

Consider now a sequence $s=\left(s_{0}, \ldots, s_{t}\right)$ of elements of $X$. It will be important to compute the expected payoffs in the subgame that begins with a move of nature following the rejection of the alternatives in the sequence $s_{0}, \ldots, s_{t}$. Notice that this subgame is isomorphic to the game $\Gamma_{s_{t}}$. We let $f[s]$ denote the continuation collective strategy after the rejection of the alternatives in $s$. It is specified by

$$
f[s](r)=f(s \oplus r), \quad r \in S,
$$

where $s \oplus r$ is the concatenation of $s$ and $r$. The expected payoffs after the rejection of the sequence $s_{0}, \ldots, s_{t}$ of alternatives are then given by $V_{s_{t}}\left(f\left[s_{0}, \ldots, s_{t}\right]\right)$.

EXAmple 6.1: Consider the profile $\sigma$ of action-independent strategies given by (4.1). The corresponding collective strategy $f_{\sigma}$ is given by $f_{\sigma}\left(s_{0}, \ldots, s_{t}\right)=1$ if and only if $s_{t} \in$ $\{\bar{x}\} \cup \operatorname{SD}(\bar{x})$. If $\bar{x}$ has the core property, then $\mathrm{SD}(\bar{x})$ is empty and $V_{x}\left(f_{\sigma}\right)=u(\bar{x})$ for every $x \in X$. This equation holds since by our assumption the Markov process $\mu$ is irreducible, so starting from any alternative $x$ the process arrives at $\bar{x}$ with non-zero probability.

For $f \in F$, we define

$$
\begin{aligned}
\mathrm{SD}(f) & =\left\{x \in X \mid\left\{i \in N: u_{i}(x)>V_{x, i}(f)\right\} \in \mathcal{C}(x)\right\}, \\
\mathrm{WD}(f) & =\left\{x \in X \mid\left\{i \in N: u_{i}(x) \geq V_{x, i}(f)\right\} \in \mathcal{C}(x)\right\} .
\end{aligned}
$$

The alternatives in $\operatorname{SD}(f)$ are said to strictly dominate the collective strategy $f$ and those in $\mathrm{WD}(f)$ are said to weakly dominate it. The alternative $x$ strictly dominates $f$ if a decisive set of players prefers accepting $x$ over rejecting it and playing the rest of the game in accordance with the collective strategy $f$. The definition of strict dominance extends the one in Section 4: The alternative $x$ strictly dominates the alternative $y$ if and only if $x$ strictly dominates the collective strategy $f$ defined by setting $f\left(s_{0}, \ldots, s_{t}\right)=1$ if and only if $s_{t}=y$. This equivalence holds because in this case $V_{x}(f)=u(y)$. 
We now present a notion of equilibrium that is in terms of collective strategies only.

Definition 6.2: The collective strategy $f \in F$ is a collective equilibrium if for every sequence $s=\left(s_{0}, \ldots, s_{t}\right) \in S$ it holds that

$$
\begin{array}{rll}
s_{t} \in \mathrm{SD}(f[s]) & \text { implies } & f(s)=1, \\
s_{t} \notin \mathrm{WD}(f[s]) & \text { implies } & f(s)=0 .
\end{array}
$$

Notice that a breakdown alternative is never rejected in a collective equilibrium as a breakdown alternative strictly dominates any collective strategy. The next result shows that a collective equilibrium induces a subgame perfect equilibrium in pure actionindependent strategies.

TheOREm 6.3: Let $f \in F$ be a collective equilibrium. Then the pure action-independent joint strategy defined for $i \in N$ and $s=\left(s_{0}, \ldots, s_{t}\right) \in S$ by

$$
\sigma_{i}(s)= \begin{cases}\mathrm{y}, & \text { if } u_{i}\left(s_{t}\right)>V_{s_{t}, i}(f[s]) \\ \mathrm{y}, & \text { if } u_{i}\left(s_{t}\right)=V_{s_{t}, i}(f[s]) \text { and } f(s)=1 \\ \mathrm{n}, & \text { otherwise, }\end{cases}
$$

is a subgame perfect equilibrium of the game $\Gamma$ with $f_{\sigma}=f$.

Proof: We verify that $f_{\sigma}=f$. Consider some $s=\left(s_{0}, \ldots, s_{t}\right) \in S$ and let $C=\{i \in N \mid$ $\left.\sigma_{i}(s)=\mathrm{y}\right\}$ be the set of players voting in favor after the sequence $s$.

In case $f(s)=1$, we have by definition of $\sigma$ that $C=\left\{i \in N \mid u_{i}\left(s_{t}\right) \geq V_{s_{t}, i}(f[s])\right\}$. By (6.3) it holds that $s_{t} \in \mathrm{WD}(f[s])$, so $C$ belongs to $\mathcal{C}\left(s_{t}\right)$. We conclude that $f_{\sigma}(s)=1$.

In case $f(s)=0$, we have by definition of $\sigma$ that $C=\left\{i \in N \mid u_{i}\left(s_{t}\right)>V_{s_{t}, i}(f[s])\right\}$. By (6.2) it holds that $s_{t} \notin \mathrm{SD}(f[s])$ which means that $C$ does not belong to $\mathcal{C}\left(s_{t}\right)$. We conclude that $f_{\sigma}(s)=0$.

It is straightforward to verify that the joint strategy $\sigma$ satisfies the one-shot deviation property. We invoke Theorem 3.1 to conclude that the joint strategy $\sigma$ is a subgame perfect equilibrium.

The next result shows that any subgame perfect equilibrium in pure action-independent strategies induces a collective equilibrium.

THEOREM 6.4: Let $\sigma$ be a subgame perfect equilibrium of $\Gamma$ in pure action-independent strategies. Then $f_{\sigma}$ is a collective equilibrium. 
Proof: Let $f=f_{\sigma}$. We show first that Condition (6.2) holds. Let $s=\left(s_{0}, \ldots, s_{t}\right) \in S$ be such that $s_{t} \in \mathrm{SD}(f[s])$. If $s_{t}$ is a breakdown alternative, then $f(s)=1$, so Condition (6.2) holds. Consider the case where $s_{t}$ is not a breakdown alternative. Suppose that $f(s)=0$. Since $s_{t} \in \mathrm{SD}(f[s])$, the set of players $C=\left\{i \in N \mid u_{i}\left(s_{t}\right)>V_{s_{t}, i}(f[s])\right\}$ belongs to $\mathcal{C}\left(s_{t}\right)$. Now label the players in $C$ as $\left\{i_{1}, \ldots, i_{m}\right\}$, where $i_{1} \prec \cdots \prec i_{m}$. For $i \in N$, let $a_{i}=\sigma_{i}(s)$ and $a=\left(a_{1}, \ldots, a_{n}\right)$. Consider the action profiles $a^{0}, a^{1}, \ldots, a^{m}$ where $a^{0}=a$ and, for every $k, 1 \leq k \leq m$, we define

$$
a_{i}^{k}= \begin{cases}\mathrm{y}, & \text { if } i \in\left\{i_{1}, \ldots, i_{k}\right\} \\ a_{i}, & \text { otherwise }\end{cases}
$$

Since by our supposition the strategy $\sigma$ results in the rejection of alternative $s_{t}$, we have $a^{0} \notin A^{*}\left(s_{t}\right)$. On the other hand, since $a_{i}^{m}=\mathrm{y}$ for every $i \in C$ and since $C \in \mathcal{C}\left(s_{t}\right)$, we have that $a^{m} \in A^{*}\left(s_{t}\right)$. Let $k^{*}$ be the least integer for which $a^{k^{*}} \in A^{*}\left(s_{t}\right)$, and let $i^{*}=i_{k^{*}}$. Notice that $\sigma_{i^{*}}(s)=a_{i^{*}}=\mathrm{n}$ for otherwise it would be the case that $a^{k^{*}-1}=a^{k^{*}}$, which contradicts the choice of $k^{*}$.

Consider now any history $h$ in $H_{i^{*}}$ where the sequence of alternatives is $s$ and the vote of players $i \prec i^{*}$ in period $t$ is equal to $a_{i}^{k^{*}}$. Since $\sigma$ is action-independent, an n-vote by player $i^{*}$ at $h$ leads to the action profile $a^{k^{*}-1}$ in period $t$ and hence to the rejection of $s_{t}$. A $\mathrm{y}$-vote by $i^{*}$ at $h$ leads to the action profile $a^{k^{*}}$ and hence to the acceptance of $s_{t}$. Since $u_{i^{*}}\left(s_{t}\right)>V_{s_{t}, i^{*}}(f[s])$, subgame perfection requires that player $i^{*}$ cast a $\mathrm{y}$-vote at $h$, whereas $\sigma_{i^{*}}(s)=\mathrm{n}$, a contradiction.

We now prove that Condition (6.3) holds. Let $s=\left(s_{0}, \ldots, s_{t}\right) \in S$ be such that $s_{t} \notin \mathrm{WD}(f[s])$. It follows that $s_{t}$ is not a breakdown alternative. Suppose that $f(s)=1$. Since $s_{t} \notin \mathrm{WD}(f[s])$, the set $C=\left\{i \in N \mid u_{i}\left(s_{t}\right)<V_{s_{t}, i}(f[s])\right\}$ has a non-empty intersection with every member of $\mathcal{C}\left(s_{t}\right)$. Now label the players in $C$ as $\left\{i_{1}, \ldots, i_{m}\right\}$, where $i_{1} \prec \cdots \prec i_{m}$. For $i \in N$, let $a_{i}=\sigma_{i}(s)$ and $a=\left(a_{1}, \ldots, a_{n}\right)$. Consider the action profiles $a^{0}, a^{1}, \ldots, a^{m}$ where $a^{0}=a$ and, for every $k, 1 \leq k \leq m$, we define

$$
a_{i}^{k}= \begin{cases}\mathrm{n}, & \text { if } i \in\left\{i_{1}, \ldots, i_{k}\right\} \\ a_{i}, & \text { otherwise }\end{cases}
$$

Since by our supposition the strategy $\sigma$ results in the acceptance of alternative $s_{t}$, we have $a^{0} \in A^{*}\left(s_{t}\right)$. On the other hand, since $a_{i}^{m}=\mathrm{n}$ for every $i \in C$ and the intersection of $C$ with every member of $\mathcal{C}\left(s_{t}\right)$ is non-empty, we have that $a^{m} \notin A^{*}\left(s_{t}\right)$. Let $k^{*}$ be the least integer for which $a^{k^{*}} \notin A^{*}\left(s_{t}\right)$, and let $i^{*}=i_{k^{*}}$. Notice that $\sigma_{i^{*}}(s)=a_{i^{*}}=\mathrm{y}$ for otherwise it would be the case that $a^{k^{*}-1}=a^{k^{*}}$, which contradicts the choice of $k^{*}$. 
Consider now any history $h$ in $H_{i^{*}}$ where the sequence of alternatives is $s$ and the vote of players $i \prec i^{*}$ in period $t$ is equal to $a_{i}^{k^{*}}$. Since $\sigma$ is action-independent, a y-vote by player $i^{*}$ at $h$ leads to the action profile $a^{k^{*}-1}$ in period $t$ and hence to the acceptance of $s_{t}$. An n-vote by $i^{*}$ at $h$ leads to the action profile $a^{k^{*}}$ and hence to the rejection of $s_{t}$. Since $u_{i^{*}}\left(s_{t}\right)<V_{s_{t}, i^{*}}(f[s])$, subgame perfection requires that player $i^{*}$ cast an n-vote at $h$, whereas $\sigma_{i^{*}}(s)=\mathrm{y}$, a contradiction.

As a consequence of Theorems 6.3 and 6.4, we can perform our analysis using collective rather than individual strategies. We refer to members of $S$ as collective histories. Individual strategies can be recovered from a given collective strategy using Equation (6.4).

\section{$7 \quad$ Simple collective strategies}

Consider a tuple $\mathcal{F}=\left(f_{j}: j \in\{0\} \cup X\right)$ consisting of the collective strategy $f_{0}$ and, for every $x \in X$, a collective strategy $f_{x}$. We define a new collective strategy $f$ as follows. Consider some sequence of alternatives $s=\left(s_{0}, \ldots, s_{t}\right) \in S$. Follow the collective strategy $f_{0}$ until the first time $t^{0}$ such that $f_{0}\left(s_{0}, \ldots, s_{t^{0}}\right)=1$. If such a $t^{0}$ does not exist or $t^{0}=t$, then define $f(s)=f_{0}(s)$. Otherwise, switch to the collective strategy $f_{s_{t^{0}}}$ and delete the history $\left(s_{0}, \ldots, s_{t^{0}}\right)$. Follow the collective strategy $f_{s_{t} 0}$ until the first time $t^{1}$ such that $f_{s_{t^{0}}}\left(s_{t^{0}+1}, \ldots, s_{t^{1}}\right)=1$. If such a $t^{1}$ does not exist or $t^{1}=t$, then define $f(s)=f_{s_{t^{0}}}(s)$. Otherwise, switch to the collective strategy $f_{s_{t^{1}}}$, and so on. Notice that switches occur after "unlawful" rejections, where instead of an acceptance a rejection has taken place.

The collective strategy $f$ thus constructed is said to be induced by the tuple $\mathcal{F}$. A collective strategy is said to be simple if it is induced by some tuple $\mathcal{F}$ of strategies. This terminology is motivated by the resemblance of our definition to that in Abreu (1988), see the discussion at the end of this section.

The formal definition of a collective strategy $f$ induced by $\mathcal{F}$ is by induction on the length of the sequence of alternatives. Define $f(x)=f_{0}(x)$ for each $x \in X$. Now suppose that $f$ has already been defined on each sequence of alternatives of length less than or equal to $t$. Consider a sequence $s=\left(s_{0}, \ldots, s_{t}\right)$ of length $t+1$. Let

$$
K(s)=\left\{k \in\{0, \ldots, t-1\} \mid f\left(s_{0}, \ldots, s_{k}\right)=1\right\}
$$

and define

$$
f\left(s_{0}, \ldots, s_{t}\right)= \begin{cases}f_{0}\left(s_{0}, \ldots, s_{t}\right), & \text { if } K(s)=\emptyset \\ f_{s_{k}}\left(s_{k+1}, \ldots, s_{t}\right), & \text { if } K(s) \neq \emptyset \text { and } k=\max K(s) .\end{cases}
$$




\begin{tabular}{|c|cccccc|}
\hline$t$ & 0 & 1 & 2 & 3 & 4 & 5 \\
\hline$s_{t}$ & $x_{1}$ & $x_{3}$ & $x_{1}$ & $x_{2}$ & $x_{1}$ & $x_{1}$ \\
\hline$f\left(s_{0}, \ldots, s_{t}\right)$ & 1 & 0 & 0 & 1 & 1 & 0 \\
\hline
\end{tabular}

Table 5: The collective strategy $f$.

The following example illustrates our definitions.

ExAmple 7.1: Let $X=\left\{x_{1}, x_{2}, x_{3}\right\}$. Consider the collective strategies $g$ and $h$ where $g(s)=1$ for every $s \in S$ and

$$
h\left(s_{0}, \ldots, s_{t}\right)= \begin{cases}1, & \text { if }\left[t=0 \text { and } s_{0}=x_{2}\right] \text { or }\left[t \geq 1 \text { and } s_{t} \in\left\{x_{2}, x_{3}\right\}\right] \\ 0, & \text { otherwise. }\end{cases}
$$

Consider now the tuple $\left(f_{0}, f_{x_{1}}, f_{x_{2}}, f_{x_{3}}\right)=(g, h, g, g)$ and let $f$ be the induced simple collective strategy. Some of the values of $f$ are given by Table 5. To derive these values, we reason as follows. Each alternative is accepted according to the collective strategy $f_{0}=g$, hence $f\left(x_{1}\right)=1$. If $x_{1}$ is rejected instead, we switch to the collective strategy $f_{x_{1}}=h$. According to $h$ only $x_{2}$ is initially accepted, so $f\left(x_{1}, x_{3}\right)=0$. After the first round, only $x_{2}$ and $x_{3}$ are accepted according to $h$, therefore $f\left(x_{1}, x_{3}, x_{1}\right)=0$ and $f\left(x_{1}, x_{3}, x_{1}, x_{2}\right)=1$. If $x_{2}$ is rejected, we switch to the collective strategy $f_{x_{2}}=g$ according to which each alternative is accepted, hence $f\left(x_{1}, x_{3}, x_{1}, x_{2}, x_{1}\right)=1$. If $x_{1}$ is rejected we switch to $f_{x_{1}}=h$. Since according to $h$ only $x_{2}$ is initially accepted we have $f\left(x_{1}, x_{3}, x_{1}, x_{2}, x_{1}, x_{1}\right)=0$.

A game-plan corresponds to that part of a collective strategy $f$ that is to be followed until the first deviation from $f$ occurs. More precisely, the game-plan $P(f)$ of the collective strategy $f$ is defined as the set of sequences $\left(s_{0}, \ldots, s_{t}\right) \in S$ such that there is no $k \in$ $\{0, \ldots, t-1\}$ with $f\left(s_{0}, \ldots, s_{k}\right)=1$. The payoffs of a collective strategy depend only on the game-plan. Formally, if $f$ and $f^{\prime}$ are collective strategies with $P(f)=P\left(f^{\prime}\right)$, then $V_{x}(f)=V_{x}\left(f^{\prime}\right)$ for each $x \in X$.

The following lemma is obvious from the definition of an induced collective strategy. It will be helpful for computing the payoffs of a simple collective strategy.

Lemma 7.2: Let the collective strategy $f$ be induced by $\mathcal{F}=\left(f_{j}: j \in\{0\} \cup X\right)$ and consider $s=\left(s_{0}, \ldots, s_{t}\right) \in S$. Then

1. $P(f)=P\left(f_{0}\right)$.

2. If $f(s)=1$, then $P(f[s])=P\left(f_{s_{t}}\right)$. 
3. If $f(s)=0$ and $K(s)=\emptyset$, then $P(f[s])=P\left(f_{0}[s]\right)$.

4. If $f(s)=0, K(s) \neq \emptyset$, and $k=\max K(s)$, then $P(f[s])=P\left(f_{s_{k}}\left[s_{k+1}, \ldots, s_{t}\right]\right)$.

In particular, the collective strategy $f$ is completely characterized by the tuple of gameplans $\left(P\left(f_{j}\right): j \in\{0\} \cup X\right)$. We have the following corollary.

Corollary 7.3: Let $\mathcal{F}=\left(f_{j}: j \in\{0\} \cup X\right)$ and $\mathcal{G}=\left(g_{j}: j \in\{0\} \cup X\right)$. If $P\left(f_{j}\right)=P\left(g_{j}\right)$ for each $j \in\{0\} \cup X$, then the collective strategy induced by $\mathcal{F}$ equals that induced by $\mathcal{G}$.

In view of this observation we shall say that the collective strategy $f$ is induced by a tuple of game-plans, rather than a tuple of collective strategies. The game-plan $P\left(f_{0}\right)=$ $P(f)$ is said to be the main game-plan of the collective strategy $f$. An unlawful rejection of an alternative $x$ triggers the punishment game-plan $P\left(f_{x}\right)$.

Notwithstanding the completely different context under consideration, our notion of simplicity bears some resemblance to the one of Abreu (1988), Definition 1. Abreu (1988) defines a simple strategy in a repeated game by a collection of paths, the main path and $n$ punishments paths, one for every player. A path is a sequence of actions in a stage game and corresponds to our concept of a game-plan. A simple strategy is then defined as follows: the main path is to be followed until the first unilateral deviation, and a unilateral deviation by player $i$ at any point in the game triggers the corresponding punishment path of play. By comparison, in our definition of simplicity, the punishment game-plan does not depend on the identity of the deviating player, but rather on what alternative has been unlawfully rejected.

\section{Two-step game-plans}

In this section we consider collective strategies that are induced by two-step game-plans. Before we proceed with the definition of a two-step game-plan, we explore the simpler, and more restrictive, condition that the collective strategy be induced by stationary gameplans.

Definition 8.1: For every $Y \subset X$, the collective strategy $f_{Y}$ is defined by setting, for every $s=\left(s_{0}, \ldots, s_{t}\right) \in S$,

$$
f_{Y}(s)= \begin{cases}1, & \text { if } s_{t} \in Y \\ 0, & \text { otherwise }\end{cases}
$$

A collective strategy $f \in F$ is stationary if $f=f_{Y}$ for some $Y \subset X$. 
The definition of a stationary collective strategy $f$ in Definition 8.1 is consistent with the definition of a stationary strategy $\sigma$ in Definition 4.1 in the sense that the pure actionindependent joint strategy $\sigma$ derived from a stationary collective strategy $f$ by means of (6.4) is stationary, and a stationary strategy $\sigma$ induces a stationary collective strategy $f_{\sigma}$. A stationary collective strategy is easily seen to be simple.

Definition 8.2: A collective strategy $f$ is induced by stationary game-plans if it is induced by a tuple $\mathcal{F}=\left(f_{j}: j \in\{0\} \cup X\right)$ where each $f_{j}$ is stationary.

Notice that a collective strategy induced by stationary game-plans need not be stationary itself. We show that the game of Example 4.6 has a collective equilibrium induced by stationary game--plans.

ExAmple 8.3: Consider Example 4.6. When $\delta \leq 1 / 2$, we have already derived that there is a subgame perfect equilibrium in pure stationary strategies where all alternatives are accepted.

We consider next the case where $\delta>1 / 2$. Consider the collective strategy $f$ induced by the tuple of game-plans

$$
\left(f_{0}, f_{x_{0}}, f_{x_{1}}, f_{x_{2}}, f_{x_{3}}\right)=\left(f_{X}, f_{X}, f_{\left\{x_{0}, x_{1}, x_{2}\right\}}, f_{\left\{x_{0}, x_{2}, x_{3}\right\}}, f_{\left\{x_{0}, x_{1}, x_{3}\right\}}\right) .
$$

Under the collective strategy $f$, every alternative is accepted in period 0 and the breakdown alternative $x_{0}$ is accepted in every period. Suppose, for some $i=1,2,3$, alternative $x_{i}$ is unlawfully rejected. The punishment game-plan $P\left(f_{x_{i}}\right)$ is such that the most attractive alternative $x_{i-1}$ for player $i$ is no longer accepted.

According to Definition 6.2, $f \in F$ is a collective equilibrium in the game of Example 4.6 if for every sequence $s=\left(s_{0}, \ldots, s_{t}\right) \in S$, where $s_{t}=x_{i}$, it holds that $f(s)=1$ if $i=0$, and for $i=1,2,3$ it holds that

$$
\begin{aligned}
& u_{i}\left(x_{i}\right)>V_{i}(f[s]) \quad \text { implies } f(s)=1, \\
& u_{i}\left(x_{i}\right)<V_{i}(f[s]) \quad \text { implies } f(s)=0 .
\end{aligned}
$$

To verify Condition (8.1), suppose $f(s)=0$. Assume first that $s_{t}=x_{1}$. This means that the punishment game-plan $P\left(f_{x_{2}}\right)$ is being followed. Since $f_{x_{2}}$ is stationary, we find that $V_{1}(f[s])=V_{1}\left(f_{x_{2}}\right)=5 \delta /(3-\delta)>1=u_{1}\left(x_{1}\right)$, where the first equality uses part 4 of Lemma 7.2 and the inequality uses the fact that $\delta>1 / 2$. The cases where $s_{t}=x_{2}$ and $s_{t}=x_{3}$ are similar.

To verify Condition (8.2), suppose $f(s)=1$. We have that $V_{i}(f[s])=V_{i}\left(f_{x_{i}}\right)=$ $\delta /(3-\delta)<1=u_{i}\left(x_{i}\right)$, where the first equation uses part 2 of Lemma 7.2. 
Example 4.6 demonstrates that the unique stationary equilibrium is in mixed strategies, has payoffs $(1,1,1)$ irrespective of the value of $\delta$, and leads to inefficiency because of delay, which tends to infinity as $\delta$ goes to 1 . The collective equilibrium constructed here has immediate acceptance and Pareto efficient payoffs $V(f)=(2 \delta, 2 \delta, 2 \delta)$.

We show next that the game of Example 4.4 admits no collective equilibrium induced by stationary game-plans. We define the carrier $\operatorname{car}(f)$ of $f$ as the set of alternatives $x \in X$ for which there exists a sequence $s=\left(s_{0}, \ldots, s_{t}\right) \in S$ such that $s_{t}=x$ and $f(s)=1$.

ExAmple 8.4: According to Definition $6.2, f \in F$ is a collective equilibrium in the game of Example 4.4 if for every sequence $s=\left(s_{0}, \ldots, s_{t}\right) \in S$, where $s_{t}=x_{i}$, it holds that

$$
\begin{aligned}
& u_{i}\left(x_{i}\right)>V_{i}(f[s]) \text { implies } f(s)=1 \text {, } \\
& u_{i}\left(x_{i}\right)<V_{i}(f[s]) \text { implies } f(s)=0 \text {. }
\end{aligned}
$$

Let $f$ be a collective equilibrium. We show that $V_{i}(f)>0$ for each player $i$. For suppose that $V_{i}(f)=0$ for some $i \in N$. We must then have $f\left(x_{i}\right)=0$ and $V_{i}\left(f\left[x_{i}\right]\right)=0$, where the first equality follows from $u_{i}\left(x_{i}\right)>0$. This contradicts Condition (8.3). For each $s \in S$, since the collective strategy $f[s]$ is a collective equilibrium, we have $V_{i}(f[s])>0$ for each $i \in N$.

The fact that $V_{i}(f)>0$ for each player $i$ implies that car $(f)$ contains at least two alternatives. We argue next that $x_{1} \in \operatorname{car}(f)$. Suppose on the contrary, so $\operatorname{car}(f)=$ $\left\{x_{2}, x_{3}\right\}$. Take any sequence $s=\left(s_{0}, \ldots, s_{t}\right) \in S$ such that $s_{t}=x_{3}$ and $f(s)=1$. Condition (8.4) implies that $u_{3}\left(x_{3}\right) \geq V_{3}(f[s])$. On the other hand since $\operatorname{car}(f[s]) \subset \operatorname{car}(f)=$ $\left\{x_{2}, x_{3}\right\}$ and the cardinality of $\operatorname{car}(f[s])$ equals 2 , it holds that $V_{3}(f[s])$ is a strictly convex combination of $u_{3}\left(x_{2}\right)$ and $u_{3}\left(x_{3}\right)$, so $V_{3}(f[s])>u\left(x_{3}\right)$, a contradiction.

Now suppose $f$ is induced by stationary game-plans. Let $f_{x_{1}}$ be given by $f_{Y}$ for some $Y \subset X$. Take any sequence $s=\left(s_{0}, \ldots, s_{t}\right) \in S$ such that $s_{t}=x_{1}$ and $f(s)=1$. We have $V(f[s])=V\left(f_{Y}\right)$. Since $V_{i}\left(f_{Y}\right)>0$ for each $i \in N$, the set $Y$ contains at least two alternatives. By Condition (8.4) we have $u_{1}\left(x_{1}\right) \geq V_{1}(f[s])=V_{1}\left(f_{Y}\right)$. This inequality, together with the fact that $Y$ contains 2 or 3 alternatives implies that $Y=\left\{x_{1}, x_{2}\right\}$ and in particular that $f\left(s \oplus x_{3}\right)=0$. We obtain a contradiction since $V_{3}\left(f\left[s \oplus x_{3}\right]\right)=V_{3}\left(f_{Y}\right)=$ $7 / 2<4=u_{3}\left(x_{3}\right)$, so by Condition (8.3) it should hold that $f\left(s \oplus x_{3}\right)=1$.

Since the game of Example 4.4 has no collective equilibrium that is induced by stationary game-plans, we now shift our attention to a bigger set of collective strategies, the one induced by two-step game-plans. 
Definition 8.5: For every $X^{1} \subset X^{2} \subset X$ and for every non-negative integer $m$, the collective strategy $f_{X^{1}, m, X^{2}}$ is defined by setting, for every $s=\left(s_{0}, \ldots, s_{t}\right) \in S$,

$$
f(s)= \begin{cases}1, & \text { if }\left[t<m \text { and } s_{t} \in X^{1}\right] \text { or }\left[t \geq m \text { and } s_{t} \in X^{2}\right] \\ 0, & \text { otherwise. }\end{cases}
$$

A collective strategy $f \in F$ is two-step if $f=f_{X^{1}, m, X^{2}}$ for some $X^{1} \subset X^{2} \subset X$ and non-negative integer $m$. The threshold of $f$ is zero if $X^{1}=X^{2}$ and is equal to the integer $m$ otherwise.

According to a two-step collective strategy, the players wait for nature to choose an alternative from $X^{1}$ in the first $m$ periods; as soon as such an alternative is chosen by nature, it is accepted. If no alternative from $X^{1}$ is chosen in the first $m$ periods, then the players wait for an alternative from the bigger set $X^{2}$.

Definition 8.6: A collective strategy $f$ is induced by two-step game-plans if it is induced by a tuple $\mathcal{F}=\left(f_{j}: j \in\{0\} \cup X\right)$ where each $f_{j}$ is two-step.

Definition 8.7: The set of collective equilibria induced by two-step game-plans is denoted by $T$.

EXAMPLE 8.8: The collective strategy $g=f_{X}$ in Example 7.1 is stationary, while the collective strategy $h=f_{\left\{x_{2}\right\}, 1,\left\{x_{2}, x_{3}\right\}}$ is two-step with a threshold of 1 . The collective strategy $f$ is therefore induced by two-step game-plans. We now show that $f$ is a collective equilibrium in the game $\Gamma$ defined in Example 4.4, so for that example the set $T$ is non-empty. We verify that $f$ satisfies Conditions (8.3) and (8.4).

Consider $s=\left(s_{0}, \ldots, s_{t}\right) \in S$ such that $f(s)=1$. Assume first that $s_{t} \in\left\{x_{2}, x_{3}\right\}$. After the rejection of $s_{t}$, the play of the game continues in accordance with the collective strategy $g$, hence $V(f[s])=V(g)=(4 / 3,11 / 3,11 / 3)$. For players $i \in\{2,3\}$, we have that $u_{i}\left(x_{i}\right)=4>11 / 3=V_{i}(f[s])$, so Condition (8.4) is satisfied. Now assume $s_{t}=x_{1}$. After the rejection of $x_{1}$, the play of the game continues in accordance with the collective strategy $h$ which results in a payoff of 1 to player 1 , thus $u_{1}\left(x_{1}\right)=1=V_{1}(h)=V_{1}(f[s])$. This shows that Condition (8.4) is satisfied.

Now take some $s=\left(s_{0}, \ldots, s_{t}\right) \in S$ such that $f(s)=0$. Notice that rejections are only prescribed by the punishment game-plan $h$. It follows that either we are in the first round of $h$ and $s_{t} \in\left\{x_{1}, x_{3}\right\}$ or we are at least in the second round of $h$ and $s_{t}=x_{1}$. In either case, the continuation play after $s$ prescribes the acceptance of alternatives $x_{2}$ and $x_{3}$ and the rejection of $x_{1}$, so $V(f[s])=(3 / 2,2,11 / 2)$. We see that $u_{1}\left(x_{1}\right)=1<3 / 2=V_{1}(f[s])$ and $u_{3}\left(x_{3}\right)=4<11 / 2=V_{3}(f[s])$. Hence $f$ satisfies Condition (8.3). 


\section{Equilibria induced by two-step game-plans}

We are now in a position to state the main result of the paper, which is that the game $\Gamma$ has a collective equilibrium $f$ induced by two-step game-plans.

THEOREM 9.1: The set $T$ is non-empty.

In addition, our construction will be such that the main game-plan is not only two-step, but even stationary.

The proof of the theorem consists of two parts. The first part can be thought of as iterated elimination of unacceptable alternatives: We inductively reduce the set of alternatives by eliminating those alternatives that cannot be accepted in any collective equilibrium that is induced by two-step game-plans. The reduction of the set of acceptable alternatives in turn results in the elimination of collective strategies that can be used as punishment game-plans. At the end of this process we are left with a set of acceptable alternatives, and, corresponding to each surviving alternative, a two-step game-plan that is a suitable punishment game-plan following an unlawful rejection of that alternative. The surviving alternatives will be called acceptable and surviving game-plans viable.

In the second part of the proof we use acceptable alternatives and viable game-plans to construct a collective equilibrium in $T$. We also show that the set of acceptable alternatives and of viable game-plans completely characterize the set $T$ of collective equilibria.

Set $Y_{0}=X$. Define $F_{0}$ to be the subset of $F$ consisting of two-step collective strategies $f$ satisfying Condition (6.2): for each $s=\left(s_{0}, \ldots, s_{t}\right) \in S$, if $s_{t} \in \mathrm{SD}(f[s])$ then $f(s)=1$. Notice that while a collective strategy in $F_{0}$ satisfies Condition (6.2) of collective equilibrium, it may well violate Condition (6.3). The set $F_{0}$ contains $f_{X}$, so is non-empty. We now give a simple criterion to check whether a two-step collective strategy satisfies Condition (6.2).

Lemma 9.2: Consider some $X^{1}, X^{2} \subset X$ with $X^{1} \subset X^{2}$. For $m=0,1, \ldots$ define the collective strategy $g_{m}=f_{X^{1}, m, X^{2}}$.

1. The collective strategy $g_{0}$ satisfies Condition (6.2) if and only if $\operatorname{SD}\left(g_{0}\right) \subset X^{2}$.

2. Consider some non-negative integer $m$. The collective strategy $g_{m+1}$ satisfies Condition (6.2) if and only if $g_{m}$ satisfies Condition (6.2) and $\operatorname{SD}\left(g_{m}\right) \subset X^{1}$.

Proof: To prove the first claim observe that $g_{0}=f_{X^{2}}$. Consider $s=\left(s_{0}, \ldots, s_{t}\right)$. It holds that $g_{0}(s)=1$ if and only if $s_{t} \in X^{2}$. Since $g_{0}[s]=g_{0}$, the result follows at once. 
To prove the second claim we notice that for each $x \in X$ it holds that $g_{m+1}[x]=g_{m}$. Moreover, for each sequence $s=\left(s_{0}, \ldots, s_{t}\right)$ with $t \geq 1$ we have $g_{m+1}(s)=g_{m}\left(s_{1}, \ldots, s_{t}\right)$ and $g_{m+1}[s]=g_{m}\left[s_{1}, \ldots, s_{t}\right]$.

To prove the "only if" part assume $g_{m+1}$ satisfies Condition (6.2). Consider some sequence $\left(s_{1}, \ldots, s_{t}\right)$. If $s_{t} \in \mathrm{SD}\left(g_{m}\left[s_{1}, \ldots, s_{t}\right]\right)$ then it holds that $s_{t} \in \mathrm{SD}\left(g_{m+1}\left[s_{0}, s_{1}, \ldots, s_{t}\right]\right)$ for every $s_{0} \in X$, so $g_{m+1}\left(s_{0}, s_{1}, \ldots, s_{t}\right)=1$ and therefore $g_{m}\left(s_{1}, \ldots, s_{t}\right)=1$. We conclude that $g_{m}$ satisfies Condition (6.2). If $x \in \mathrm{SD}\left(g_{m}\right)$, then it holds that $x \in \operatorname{SD}\left(g_{m+1}[x]\right)$, so $g_{m+1}(x)=1$ and therefore $x \in X^{1}$.

We now prove the "if" part. Consider some $s=\left(s_{0}, \ldots, s_{t}\right) \in S$ such that $s_{t} \in$ $\mathrm{SD}\left(g_{m+1}[s]\right)$. If $t=0$, then it holds that $s_{0} \in \mathrm{SD}\left(g_{m}\right)$, hence $s_{0} \in X^{1}$, and therefore $g_{m+1}\left(s_{0}\right)=1$. If $t \geq 1$, then it holds that $s_{t} \in \mathrm{SD}\left(g_{m}\left[s_{1}, \ldots, s_{t}\right]\right)$. Since $g_{m}$ satisfies (6.2) we have $g_{m}\left(s_{1}, \ldots, s_{t}\right)=1$ and therefore $g_{m+1}(s)=1$.

For every $k \in \mathbb{N}$, we inductively define

$$
\begin{aligned}
& Y_{k}=\bigcup_{f \in F_{k-1}} \operatorname{WD}(f), \\
& F_{k}=\left\{f \in F_{0} \mid \operatorname{car}(f) \subset Y_{k}\right\} .
\end{aligned}
$$

The alternatives for which there is a suitable punishment game-plan in $F_{k-1}$, i.e. those alternatives $y$ for which there is $f \in F_{k-1}$ such that $y \in \mathrm{WD}(f)$, are collected in the set $Y_{k}$. Next the set $F_{k}$ is defined as those collective strategies $f_{X^{1}, m, X^{2}}$ in $F_{0}$ where alternatives outside $Y_{k}$ are never accepted, so both $X^{1}$ and $X^{2}$ are subsets of $Y_{k}$. From there one defines the set $Y_{k+1}$, and so on.

Lemma 9.3: For every $k \in \mathbb{N}$ it holds that $Y_{k} \subset Y_{k-1}$ and $F_{k} \subset F_{k-1}$.

Proof: The proof is by induction on $k$. It is clear that $Y_{1} \subset Y_{0}$ and $F_{1} \subset F_{0}$.

Assume for some $k \in \mathbb{N}$ we have shown that $Y_{k} \subset Y_{k-1}$ and $F_{k} \subset F_{k-1}$. We complete the proof by showing that $Y_{k+1} \subset Y_{k}$ and $F_{k+1} \subset F_{k}$.

If $y \in Y_{k+1}$, then there is $f \in F_{k}$ such that $y \in \mathrm{WD}(f)$. In view of the induction hypothesis, we have $f \in F_{k-1}$ and hence $y \in Y_{k}$. This proves that $Y_{k+1} \subset Y_{k}$. If $f \in F_{k+1}$, then $\operatorname{car}(f) \subset Y_{k+1}$. By the previous step, we have $\operatorname{car}(f) \subset Y_{k}$, so $f \in F_{k}$. This shows that $F_{k+1} \subset F_{k}$.

We define the sets

$$
Y_{*}=\bigcap_{k=0}^{\infty} Y_{k} \text { and } F_{*}=\bigcap_{k=0}^{\infty} F_{k} .
$$


Since $Y_{0}, Y_{1}, \ldots$ is a decreasing sequence of finite sets, we have $Y_{k}=Y_{k+1}=Y_{*}$ for $k$ sufficiently large, and therefore $F_{k}=F_{k+1}=F_{*}$. It follows that

$$
\begin{aligned}
& Y_{*}=\bigcup_{f \in F_{*}} \operatorname{WD}(f), \\
& F_{*}=\left\{f \in F_{0} \mid \operatorname{car}(f) \subset Y_{*}\right\} .
\end{aligned}
$$

The alternatives in $Y_{*}$ are said to be acceptable and the strategies in $F_{*}$ are said to be viable. Corresponding to each acceptable alternative $y \in Y_{*}$ there is a game-plan in $F_{*}$ that could be used as a punishment game-plan following an unlawful rejection of $y$. As follows from Theorems 9.7 and 9.9 below, the set $Y_{*}$ consists precisely of those alternatives that are accepted in some collective equilibrium $f \in T$, and $F_{*}$ consists precisely of those game-plans that can be played as the main or a punishment game-plan of such equilibria. Our aim is to show the non-emptiness of the set $Y_{*}$.

The following lemma states an important continuity property of the payoff functions. Essential is our assumption that the Markov process $\mu$ is irreducible as well as the condition that the set $X^{1}$ be non-empty.

Lemma 9.4: Consider $X^{1}, X^{2} \subset X$ with $\emptyset \neq X^{1} \subset X^{2}$. For every $x \in X$ it holds that $V_{x}\left(f_{X^{1}, m, X^{2}}\right) \longrightarrow V_{x}\left(f_{X^{1}}\right)$ as $m \rightarrow \infty$.

Proof: Fix some $i \in N$ and consider the recursive equations

$$
\begin{aligned}
V_{x, i}\left(f_{X^{1}, m+1, X^{2}}\right) & =\sum_{y \in X^{1}} \mu(y \mid x) u_{i}(y)+\sum_{y \in X \backslash X^{1}} \mu(y \mid x) V_{y, i}\left(f_{X^{1}, m, X^{2}}\right), \\
V_{x, i}\left(f_{X^{1}}\right) & =\sum_{y \in X^{1}} \mu(y \mid x) u_{i}(y)+\sum_{y \in X \backslash X^{1}} \mu(y \mid x) V_{y, i}\left(f_{X^{1}}\right) .
\end{aligned}
$$

We define $v_{x, m}=V_{x, i}\left(f_{X^{1}, m, X^{2}}\right)-V_{x, i}\left(f_{X^{1}}\right)$. It is sufficient to show that for every $x \in X \backslash X^{1}$, $v_{x, m} \rightarrow 0$ as $m \rightarrow \infty$. Subtracting equation (9.2) from (9.1) yields

$$
v_{x, m+1}=\sum_{y \in X \backslash X^{1}} \mu(y \mid x) v_{y, m}
$$

We define the column vector $v_{m}=\left(v_{y, m}\right)_{y \in X \backslash X^{1}}$. Then (9.3) can be written as $v_{m+1}=A v_{m}$, where the entry of the non-negative matrix $A$ corresponding to row $x$ and column $y$ is $\mu(y \mid x)$. The sum of the entries in any given row of $A$ is at most 1 . Moreover, we can write $A$ in upper-triangular block form, with each block irreducible and the sum of the entries in at least one row of the block strictly smaller than 1 . It then follows that the spectral radius of $A$ is smaller than 1. This in turn implies that $A^{m} \rightarrow 0$ if $m \rightarrow \infty$, see Corollary 6.2.28 and Theorem 5.6.12 of Horn and Johnson (2007), and therefore that $v_{m}=A^{m} v_{0} \rightarrow 0$ if 
$m \rightarrow \infty$

We are ready for the crucial step towards the proof of our main result: proving that the sets $Y_{k}$ are all non-empty.

THEOREM 9.5: The set $Y_{k}$ is non-empty and $f_{Y_{k}} \in F_{k}$.

Proof: The proof is by induction on $k$.

It clearly holds that the set $Y_{0}=X$ is non-empty and $f_{Y_{0}} \in F_{0}$. Assume that for some non-negative integer $k$ we have shown that $Y_{k} \neq \emptyset$ and $f_{Y_{k}} \in F_{k}$.

We prove first that $Y_{k+1} \neq \emptyset$. Suppose on the contrary that $Y_{k+1}=\emptyset$. Let $x$ be an element of $Y_{k}$ and let $g_{m}=f_{\{x\}, m, Y_{k}}$.

We claim that $g_{0}, g_{1}, \ldots$ are elements of $F_{k}$. By the induction hypothesis it holds that $g_{0}=f_{Y_{k}} \in F_{k}$. Assume that for some $m$ we have shown that $g_{m} \in F_{k}$. Then $\mathrm{SD}\left(g_{m}\right) \subset \mathrm{WD}\left(g_{m}\right) \subset Y_{k+1}=\emptyset$. Applying Lemma 9.2 we conclude that $g_{m+1}$ satisfies Condition (6.2), so is an element of $F_{0}$. Clearly $g_{m+1}$ is carried by the set $Y_{k}$, hence is an element of $F_{k}$.

For every $y \in X$ it holds that the sequence $V_{y}\left(g_{m}\right)$ approaches $V_{y}\left(f_{\{x\}}\right)$ as $m \rightarrow \infty$. Therefore, since $X$ is finite, there is $M$ sufficiently large such that $\operatorname{SD}\left(f_{\{x\}}\right) \subset \operatorname{SD}\left(g_{M}\right)$. The alternative $x$ does not have the core property, for otherwise it would be an element of $Y_{*}$ by Theorem 9.11 and hence an element of $Y_{k+1}$ which is empty by supposition. Hence the set $\mathrm{SD}(x)$ is non-empty. Since $\mathrm{SD}(x)=\mathrm{SD}\left(f_{\{x\}}\right)$, we have the inclusions

$$
\mathrm{SD}\left(f_{\{x\}}\right) \subset \mathrm{SD}\left(g_{M}\right) \subset \mathrm{WD}\left(g_{M}\right) \subset Y_{k+1},
$$

where the rightmost inclusion holds because $g_{M} \in F_{k}$. But this contradicts our supposition that $Y_{k+1}=\emptyset$.

We have thus proven that $Y_{k+1}$ is non-empty.

We show next that $f_{Y_{k+1}} \in F_{k+1}$. Let $g_{m}=f_{Y_{k+1}, m, Y_{k}}$.

We claim that $g_{0}, g_{1}, \ldots$ are elements of $F_{k}$. Indeed, $g_{0}=f_{Y_{k}} \in F_{k}$ by the induction hypothesis. Assume that for some $m$ we have shown that $g_{m} \in F_{k}$. Then it holds that $\mathrm{SD}\left(g_{m}\right) \subset \mathrm{WD}\left(g_{m}\right) \subset Y_{k+1}$. Applying Lemma 9.2.2, we conclude that $g_{m+1} \in F_{0}$. Since $\operatorname{car}\left(g_{m+1}\right)=Y_{k}, g_{m+1}$ is an element of $F_{k}$.

For every $y \in X$ it holds that the sequence $V_{y}\left(g_{m}\right)$ converges to $V_{y}\left(f_{Y_{k+1}}\right)$ as $m \rightarrow \infty$. Therefore, since $X$ is finite, there is $M$ sufficiently large such that $\operatorname{SD}\left(f_{Y_{k+1}}\right) \subset \mathrm{SD}\left(g_{M}\right)$. Now we have the inclusions

$$
\mathrm{SD}\left(f_{Y_{k+1}}\right) \subset \mathrm{SD}\left(g_{M}\right) \subset \mathrm{WD}\left(g_{M}\right) \subset Y_{k+1}
$$


where the rightmost inclusion follows since $g_{M} \in F_{k}$. By Lemma 9.2.1 it follows that $f_{Y_{k+1}} \in F_{k+1}$, as desired.

This completes the induction step.

Corollary 9.6 follows immediately from Theorem 9.5.

Corollary 9.6: The set $Y_{*}$ is non-empty and $f_{Y_{*}} \in F_{*}$.

We now construct a collective equilibrium that satisfies the properties required in Theorem 9.1. For each $x \in Y_{*}$, choose some $f_{x} \in F_{*}$ with $x \in \mathrm{WD}\left(f_{x}\right)$, and for $x \in X \backslash Y_{*}$ let $f_{x}$ be any element of $F_{*}$. As a special case we can take $f_{x}=f_{Y_{*}}$ for $x \in X \backslash Y_{*}$. Let $f_{0}$ be any element of $F_{*}$. As a special case we can take $f_{0}$ to be equal to $f_{Y_{*}}$. The set of all collective strategies induced by a tuple $\left(f_{j}: j \in\{0\} \cup X\right)$ as constructed in this paragraph is denoted by $G$.

THEOREM 9.7: Every collective strategy $f_{*} \in G$ is a collective equilibrium.

Proof: To prove that $f_{*}$ satisfies Condition (6.3) take an $s=\left(s_{0}, \ldots, s_{t}\right) \in S$ with $f_{*}(s)=$ 1. By Lemma 7.2 we have that $P\left(f_{*}[s]\right)=P\left(f_{s_{t}}\right)$ and therefore $\mathrm{WD}\left(f_{*}[s]\right)=\mathrm{WD}\left(f_{s_{t}}\right)$. Since $s_{t}$ is an element of $\operatorname{WD}\left(f_{s_{t}}\right)$ by the choice of $f_{s t}$, we conclude that Condition (6.3) is satisfied.

To prove that $f_{*}$ satisfies Condition (6.2) take an $s=\left(s_{0}, \ldots, s_{t}\right) \in S$ with $f_{*}(s)=0$. Assume first that $f_{*}\left(s_{0}, \ldots, s_{k}\right)=0$ for every $k=0, \ldots, t-1$. We have that $f_{*}(s)=f_{0}(s)$, $P\left(f_{*}[s]\right)=P\left(f_{0}[s]\right)$, and consequently $\mathrm{SD}\left(f_{*}[s]\right)=\mathrm{SD}\left(f_{0}[s]\right)$. Since $f_{0}$ satisfies Condition (6.2), $s_{t}$ is not an element of $\operatorname{SD}\left(f_{0}[s]\right)$, hence also not of $\operatorname{SD}\left(f_{*}[s]\right)$, as desired.

Assume next that $f_{*}\left(s_{0}, \ldots, s_{k}\right)=1$ for some $k=0, \ldots, t-1$. Let $k^{*}$ be the largest $k$ with this property. We have that $f_{*}(s)=f_{s_{k_{*}}}\left(s_{k^{*}+1}, \ldots, s_{t}\right), \quad P\left(f_{*}[s]\right)=$ $P\left(f_{s_{k^{*}}}\left[s_{k^{*}+1}, \ldots, s_{t}\right]\right)$, and $\mathrm{SD}\left(f_{*}[s]\right)=\mathrm{SD}\left(f_{s_{k^{*}}}\left[s_{k^{*}+1}, \ldots, s_{t}\right]\right)$. Since $f_{s_{k^{*}}}$ satisfies Condition (6.2), $s_{t}$ is not an element of $\operatorname{SD}\left(f_{s_{k^{*}}}\left[s_{k^{*}+1}, \ldots, s_{t}\right]\right)$, hence also not of $\operatorname{SD}\left(f_{*}[s]\right)$, as desired.

This completes the proof of Theorem 9.1.

In what follows we show that every collective equilibrium induced by two-step strategies can be constructed as in the paragraph preceding Theorem 9.7. More precisely, consider an element $f \in T$, a collective equilibrium induced by a collection of two-step game-plans $\left(f_{j}: j \in\{0\} \cup X\right)$. As Theorem 9.9 shows, the main game-plan of $f$ as well as all relevant punishment game-plans $f_{x}$ are elements of the set $F_{*}$. Here a punishment game-plan $f_{x}$ is relevant if $x$ belongs to the carrier of $f$. Indeed, if $x$ does not belong to the carrier 
of $f$, then the alternative $x$ is never accepted under the strategy $f$, and consequently the punishment game-plan $f_{x}$ does not play any role.

We need a preliminary lemma.

Lemma 9.8: Let $f$ be induced by the tuple $\left(f_{j}: j \in\{0\} \cup X\right)$ of two-step strategies. For every $j \in\{0\} \cup \operatorname{car}(f)$, it holds that $\operatorname{car}\left(f_{j}\right) \subset \operatorname{car}(f)$.

Proof: Let $f_{j}=f_{X^{1}, m, X^{2}}$, so $\operatorname{car}\left(f_{j}\right)=X^{2}$. We have to show that $X^{2} \subset \operatorname{car}(f)$. If $j=0$, then let $s$ be the empty sequence. If $j \in \operatorname{car}(f)$, then let $s=\left(s_{0}, \ldots, s_{t}\right) \in S$ be a sequence such that $s_{t}=j$ and $f(s)=1$. Take some $y \in X^{2}$. If $y \in X^{1}$, then $f\left(s_{0}, \ldots, s_{t}, y\right)=f_{j}(y)=1$. If $y \in X^{2} \backslash X^{1}$, then consider the sequence $\left(s_{0}, \ldots, s_{t}, y, \ldots, y\right)$ where $y$ is repeated $m+1$ times. We have $f\left(s_{0}, \ldots, s_{t}, y, \ldots, y\right)=f_{j}(y, \ldots, y)=1$. In either case it holds that $y \in \operatorname{car}(f)$.

TheOREM 9.9: Let $f$ be induced by the tuple $\left(f_{j}: j \in\{0\} \cup X\right)$ of two-step strategies. If $f$ is a collective equilibrium, then $\operatorname{car}(f) \subset Y_{*}$ and for every $j \in\{0\} \cup \operatorname{car}(f)$ it holds that $f_{j} \in F_{*}$.

Proof: We show first that $f_{0} \in F_{0}$. Let $f_{0}=f_{X^{1}, m, X^{2}}$.

Suppose there is $s=\left(s_{0}, \ldots, s_{t}\right) \in S$ such that $s_{t} \in \operatorname{SD}\left(f_{0}[s]\right)$ and $f_{0}(s)=0$. Then either $t<m$ and $s_{t} \notin X^{1}$ or $t \geq m$ and $s_{t} \notin X^{2}$. Consider the sequence $s^{\prime}=\left(s_{0}^{\prime}, \ldots, s_{t}^{\prime}\right)$ where for every $k \in\{0, \ldots, t\}$ it holds that $s_{k}^{\prime}=s_{t}$. For every $k \in\{0, \ldots, t\}$ it holds that $f_{0}\left(s_{0}^{\prime}, \ldots, s_{k}^{\prime}\right)=0$ and consequently also $f\left(s_{0}^{\prime}, \ldots, s_{k}^{\prime}\right)=0$. Therefore, $\operatorname{SD}\left(f_{0}[s]\right)=$ $\mathrm{SD}\left(f_{0}\left[s^{\prime}\right]\right)=\mathrm{SD}\left(f\left[s^{\prime}\right]\right)$, where the first equality holds since $f_{0}[s]=f_{0}\left[s^{\prime}\right]$ and the second one is implied by Lemma 7.2, case 3. Since $f$ satisfies condition $(6.2)$ and $f\left(s^{\prime}\right)=0$ we must have $s_{t} \notin \mathrm{SD}\left(f\left[s^{\prime}\right]\right)$ and consequently $s_{t} \notin \mathrm{SD}\left(f_{0}[s]\right)$, yielding a contradiction.

It follows immediately from Definition 6.2 that for every $x \in \operatorname{car}(f)$ the collective strategy induced by $f_{x}$ as the main game-plan and punishment game-plans $\left(f_{y}: y \in X\right)$ is a collective equilibrium. The result of the preceding paragraph therefore applies to show that $f_{x} \in F_{0}$ for every $x \in \operatorname{car}(f)$.

Assume we have shown that $\operatorname{car}(f) \subset Y_{k}$ and for every $j \in\{0\} \cup \operatorname{car}(f)$ it holds that $f_{j} \in F_{k}$. Consider some $x \in \operatorname{car}(f)$. Let $s=\left(s_{0}, \ldots, s_{t}\right) \in S$ be such that $s_{t}=x$ and $f(s)=1$. Since $f$ is a collective equilibrium, it holds by Condition 6.3 that $x \in \operatorname{WD}(f[s])$. Lemma 7.2, case 2, applies to show that $\mathrm{WD}(f[s])=\mathrm{WD}\left(f_{x}\right)$. Since $f_{x} \in F_{k}$ by the induction hypothesis, we have $x \in Y_{k+1}$. We have shown that $\operatorname{car}(f) \subset Y_{k+1}$. The preceding lemma now implies that $\operatorname{car}\left(f_{j}\right) \subset Y_{k+1}$ for every $j \in\{0\} \cup \operatorname{car}(f)$. It follows that $f_{j} \in F_{k+1}$. The induction step is complete. 
As a corollary of Theorem 9.9, we find that $Y_{*}$ contains all stationary collective equilibria.

COROLlary 9.10: If the collective equilibrium $f_{Y}$ is stationary, then $Y \subset Y_{*}$ and $f_{Y} \in F_{*}$.

By Theorem 4.2 and Theorem 6.4 it holds that if an alternative $x$ has the core property, then $f_{\{x\}}$ is a collective equilibrium. We thus obtain the following corollary.

Corollary 9.11: The core is a subset of $Y_{*}$.

Combining Theorems 9.7 and 9.9 provides a characterization of collective equilibria induced by two-step game-plans. Stated succinctly, this characterization is as follows.

Corollary 9.12: It holds that $G=T$.

The collection of all two-step game-plans is a countable set. Our next step is to provide an upper bound on the threshold of two-step game-plans that is sufficient to demonstrate the existence of a collective equilibrium. This restriction of the threshold leads to a finite set of two-step game-plans.

Lemma 9.13: There exists a natural number $M$ such that for all subsets $X^{1}, X^{2}$ of $X$ with $\emptyset \neq X^{1} \subset X^{2}$ it holds that $\mathrm{SD}\left(f_{X^{1}}\right) \subset \mathrm{SD}\left(f_{X^{1}, M, X^{2}}\right)$.

Proof: We explicitly define the number $M$ which has the desired property. Let

$$
\bar{u}=\max \left\{u_{i}(x) \mid i \in N, x \in X\right\}
$$

be the maximum utility level reached by any alternative. By our assumption that there is at least one alternative with a strictly positive payoff for some player, we have $\bar{u}>0$. Define

$$
\epsilon=\min \left\{u_{i}(x)-V_{x, i}\left(f_{Y}\right) \mid i \in N, x \in X, Y \subset X, \text { and } u_{i}(x)-V_{x, i}\left(f_{Y}\right)>0\right\} .
$$

Notice that $\epsilon$ is well-defined and is positive since the sets $N$ and $X$ are finite.

For $x \in X$, let $M_{x}$ be the least natural number such that for every alternative $y \in X$ there is probability greater than $1-\epsilon / \bar{u}$ that $y$ is selected at least once in the next $M-1$ rounds if the current alternative is $x$. The irreducibility of $\mu$ implies that such an $M_{x}$ exists. We define $M=\max _{x \in X} M_{x}$.

Since $f_{X^{1}}$ and $f_{X^{1}, M, X^{2}}$ coincide in periods $0, \ldots, M-1$, and the probability that period $M$ is reached conditional on a rejection of $x$ in period 0 is less than $\epsilon / \bar{u}$ under the collective strategy $f_{X^{1}}$, for every $i \in N$ we have that

$$
\left|V_{x, i}\left(f_{X^{1}, M, X^{2}}\right)-V_{x, i}\left(f_{X^{1}}\right)\right|<\left(1-\frac{\epsilon}{\bar{u}}\right) 0+\frac{\epsilon}{\bar{u}} \bar{u}=\epsilon .
$$


Now take $x \in \operatorname{SD}\left(f_{X^{1}}\right)$ and let $C=\left\{i \in N \mid u_{i}(x)>V_{x, i}\left(f_{X^{1}}\right)\right\}$. Then we have $u_{i}(x)-V_{x, i}\left(f_{X^{1}}\right) \geq \epsilon$ for every $i \in C$ by definition of $\epsilon$. Since $V_{x, i}\left(f_{X^{1}}\right)>V_{x, i}\left(f_{X^{1}, M, X^{2}}\right)-\epsilon$, we conclude that $u_{i}(x)>V_{x, i}\left(f_{X^{1}, M, X^{2}}\right)$ for every $i \in C$, so $x \in \operatorname{SD}\left(f_{X^{1}, M, X^{2}}\right)$, as desired.

We define $\bar{F}_{0}$ as the subset of $F_{0}$ consisting of two-step collective strategies with threshold at most $M$. Starting from $\bar{F}_{0}$ we inductively define

$$
\begin{aligned}
& \bar{Y}_{k}=\bigcup_{f \in F_{k-1}} \mathrm{WD}(f), \\
& \bar{F}_{k}=\left\{f \in \bar{F}_{0} \mid \operatorname{car}(f) \subset Y_{k}\right\} .
\end{aligned}
$$

We define the sets

$$
\bar{Y}_{*}=\bigcap_{k=0}^{\infty} \bar{Y}_{k} \text { and } \bar{F}_{*}=\bigcap_{k=0}^{\infty} \bar{F}_{k} .
$$

It can be shown as before that $\bar{Y}_{k}=\bar{Y}_{k+1}=\bar{Y}_{*}$ for $k$ sufficiently large, $\bar{F}_{k}=\bar{F}_{k+1}=\bar{F}_{*}$, and

$$
\begin{aligned}
& \bar{Y}_{*}=\bigcup_{f \in \bar{F}_{*}} \mathrm{WD}(f), \\
& \bar{F}_{*}=\left\{f \in \bar{F}_{0} \mid \operatorname{car}(f) \subset \bar{Y}_{*}\right\},
\end{aligned}
$$

where $\bar{Y}_{*} \neq \emptyset$. Indeed, the only adjustment in the proof occurs in the proof of Theorem 9.5, where we can twice avoid the limit argument that is used in claiming the existence of a suitable $g_{M}$, and use directly the number $M$ as coming from Lemma 9.13.

\section{Three alternatives}

In this section we consider the special case of the model with three alternatives, where the alternatives give rise to a Condorcet cycle: $\operatorname{SD}\left(x_{2}\right)=\left\{x_{1}\right\}, \operatorname{SD}\left(x_{3}\right)=\left\{x_{2}\right\}$, and $\mathrm{SD}\left(x_{1}\right)=\left\{x_{3}\right\}$. We do not make any further assumption regarding the number of players, the collection of decisive coalitions, or the Markov process by which alternatives are selected. Example 4.4 provides an illustration for the case of a quitting game with three players and time and history independent probabilities to select alternatives, and Example 4.5 for the case when decision making takes place by means of majority voting.

The next result claims that the set $Y_{*}$ as constructed in the previous section consists of all three alternatives. The equilibrium of Theorem 9.7 with $f_{0}=f_{Y_{*}}$ therefore has the immediate acceptance property: whatever alternative is chosen by nature in period zero is accepted. 
Theorem 10.1: Let $X=\left\{x_{1}, x_{2}, x_{3}\right\}$ and assume that $\operatorname{SD}\left(x_{2}\right)=\left\{x_{1}\right\}, \operatorname{SD}\left(x_{3}\right)=\left\{x_{2}\right\}$, and $\operatorname{SD}\left(x_{1}\right)=\left\{x_{3}\right\}$. Then $Y_{*}=X$.

Proof: It holds by definition that $Y_{0}=X$. We show that $Y_{1}=X$ as well, from which it follows that $Y_{k}=X$ for every $k=0,1, \ldots$

We prove first that $x_{1} \in Y_{1}$. Since $Y_{0}=X$, it clearly holds that $f_{X} \in F_{0}$.

Assume $x_{1} \in \mathrm{WD}\left(f_{X}\right)$. We then obviously have $x_{1} \in Y_{1}$.

Assume $x_{1} \notin \mathrm{WD}\left(f_{X}\right)$. Choose $M$ as in Lemma 9.13. For $m=0, \ldots, M$, we define the collective strategy $g_{m}=f_{\left\{x_{2}\right\}, m,\left\{x_{2}, x_{3}\right\}}$. It holds that $x_{1} \in \mathrm{SD}\left(x_{2}\right)=\operatorname{SD}\left(f_{\left\{x_{2}\right\}}\right)$. By the choice of the threshold $M$ we know that $\mathrm{SD}\left(f_{\left\{x_{2}\right\}}\right) \subset \mathrm{SD}\left(g_{M}\right)$ and hence $x_{1} \in \operatorname{SD}\left(g_{M}\right) \subset$ $\mathrm{WD}\left(g_{M}\right)$. Now let $k$ be the smallest number in $\{0, \ldots, M\}$ such that $x_{1} \in \mathrm{WD}\left(g_{k}\right)$.

We claim that $g_{0}, \ldots, g_{k}$ belong to $F_{0}$. To prove this we only need to show that $g_{0}, \ldots, g_{k}$ all satisfy Condition (6.2).

To show that $g_{0}=f_{\left\{x_{2}, x_{3}\right\}}$ satisfies Condition (6.2), we have to prove that $x_{1} \notin$ $\mathrm{SD}\left(f_{\left\{x_{2}, x_{3}\right\}}\right)$. We have that

$$
\begin{aligned}
V_{x_{1}}\left(f_{\left\{x_{2}, x_{3}\right\}}\right) & =\frac{\mu\left(x_{2} \mid x_{1}\right)}{1-\mu\left(x_{1} \mid x_{1}\right)} u\left(x_{2}\right)+\frac{\mu\left(x_{3} \mid x_{1}\right)}{1-\mu\left(x_{1} \mid x_{1}\right)} u\left(x_{3}\right), \\
V_{x_{1}}\left(f_{X}\right) & =\mu\left(x_{1} \mid x_{1}\right) u\left(x_{1}\right)+\mu\left(x_{2} \mid x_{1}\right) u\left(x_{2}\right)+\mu\left(x_{3} \mid x_{1}\right) u\left(x_{3}\right) .
\end{aligned}
$$

Rearranging terms we find that $u_{i}\left(x_{1}\right) \geq V_{x_{1}, i}\left(f_{\left\{x_{2}, x_{3}\right\}}\right)$ if and only if $u_{i}\left(x_{1}\right) \geq V_{x_{1}, i}\left(f_{X}\right)$. Since $x_{1} \notin \mathrm{WD}\left(f_{X}\right)$, we have $x_{1} \notin \mathrm{WD}\left(f_{\left\{x_{2}, x_{3}\right\}}\right) \supset \mathrm{SD}\left(f_{\left\{x_{2}, x_{3}\right\}}\right)$, as desired.

Assume $g_{m}$ satisfies Condition (6.2) for some integer $m \in\{0, \ldots, k-1\}$. We know that $x_{1} \notin \mathrm{SD}\left(g_{m}\right)$ by the choice of $k$ and since $m<k$. We claim that also $x_{3} \notin \operatorname{SD}\left(g_{m}\right)$. Indeed, $V_{x_{3}}\left(g_{m}\right)$ is a convex combination of $u\left(x_{2}\right)$ and $u\left(x_{3}\right)$. Hence if $u_{i}\left(x_{3}\right)>V_{x_{3}, i}\left(g_{m}\right)$ then it holds necessarily that $u_{i}\left(x_{3}\right)>u_{i}\left(x_{2}\right)$. Since $x_{3} \notin \mathrm{SD}\left(x_{2}\right)$ we conclude that $x_{3} \notin \operatorname{SD}\left(g_{m}\right)$. We have shown that $\mathrm{SD}\left(g_{m}\right) \subset\left\{x_{2}\right\}$. By Lemma 9.2.2 it follows that $g_{m+1}$ satisfies Condition (6.2).

We have thus shown that $g_{k} \in F_{0}$. Since $k$ is chosen such that $x_{1} \in \mathrm{WD}\left(g_{k}\right)$, it follows that $x_{1} \in Y_{1}$.

It follows by symmetry that $x_{2}$ and $x_{3}$ belong to $Y_{1}$.

When we apply the construction in the proof of Theorem 10.1 to Example 4.4, we find the collective strategy as defined in Example 7.1. The main game-plan is determined by the stationary collective strategy $f_{0}=f_{X}$ so all alternatives are accepted in period 0 . Since $\mathrm{WD}\left(f_{X}\right)=\left\{x_{2}, x_{3}\right\}$, the punishment game-plans following unlawful rejections of $x_{2}$ and $x_{3}$ are given by $f_{x_{2}}=f_{x_{3}}=f_{X}$. If we define $g_{m}=f_{\left\{x_{2}\right\}, m,\left\{x_{2}, x_{3}\right\}}$, then the smallest value for $m$ for which $x_{1} \in \mathrm{WD}\left(g_{m}\right)$ is equal to 1 . This leads to the choice $f_{x_{1}}=g_{1}$ in Example 7.1. 


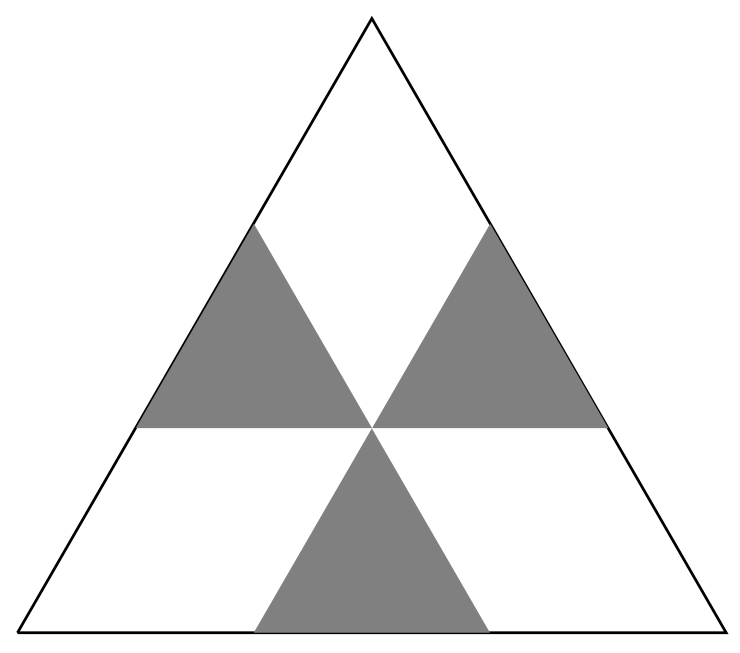

Figure 1: The set $X_{*}$.

\section{Redistributive politics}

In this section we consider a situation of redistributive politics, where three players have to allocate $K$ identical units of an indivisible good, using the simple majority voting rule. This application corresponds to the discrete version of the famous model of Baron and Ferejohn (1989). The set of alternatives is

$$
X=\left\{x \in \mathbb{N}_{0}^{3} \mid x_{1}+x_{2}+x_{3}=K\right\},
$$

where $\mathbb{N}_{0}$ is the set of natural numbers including zero, and the utility functions are given by $u_{i}(x)=x_{i}$. For every $x \in X$, the set $\mathcal{C}(x)=\{\{1,2\},\{1,3\},\{2,3\},\{1,2,3\}\}$ consists of all sets containing at least two players. We assume that each alternative is selected with equal probability, thus $\mu^{0}(y)=\mu(y \mid x)=1 /|X|$ for all $x, y \in X$. The core of this game is empty whenever $K \geq 4$.

Let $X_{*}$ denote the set of allocations where at least two players receive at least $K / 3$ units of the good, that is

$$
X_{*}=\left\{x \in X||\left\{i \in N: x_{i} \geq K / 3\right\} \mid \geq 2\right\} .
$$

The set $X_{*}$ is depicted in Figure 1. It is non-empty if $K \geq 2$. The set $X_{*}$ is symmetric, which implies $V\left(f_{X_{*}}\right)=(K / 3, K / 3, K / 3)$. It follows that $X_{*}=\mathrm{WD}\left(f_{X_{*}}\right)$, so we have the following result.

TheOrem 11.1: Assume $K \geq 2$. It holds that $f_{X_{*}}$ is a stationary collective equilibrium.

All players expect a payoff of $K / 3$ and accept any alternative which promises at least $K / 3$. It follows that the first alternative drawn which provides at least two players with a payoff greater than or equal to $K / 3$ will be accepted. 
The question we are interested in is how the set $Y_{*}$ of alternatives constructed in Section 9 relates to $X_{*}$. By Corollary 9.10 we have that $X_{*} \subset Y_{*}$. We can easily prove that the set $Y_{*}$ does not include allocations where one player gets everything.

Lemma 11.2: Assume $K \geq 3$. The alternatives $(K, 0,0),(0, K, 0)$, and $(0,0, K)$ do not belong to $Y_{*}$.

Proof: To obtain a contradiction suppose that $(K, 0,0)$ is an element of $Y_{*}$. Then there is a collective strategy $f=f_{X^{1}, m, X^{2}} \in F_{*}$ such that $V_{2}(f)=0$ or $V_{3}(f)=0$. Without loss of generality, assume $V_{3}(f)=0$. It follows that $x_{3}=0$ for each $x \in X^{2}$. In particular, the allocation $(K-1,0,1)$ is not in $X^{2}$, and since $f$ satisfies Condition $(6.2),(K-1,0,1)$ does not strictly dominate $f_{X^{2}}$. Since $V_{3}\left(f_{X^{2}}\right)=0$ this implies that $V_{1}\left(f_{X^{2}}\right) \geq K-1$. Similarly, $(0, K-1,1)$ is not in $X^{2}$, so $V_{2}\left(f_{X^{2}}\right) \geq K-1$. Adding these inequalities yields $V_{1}\left(f_{X^{2}}\right)+V_{2}\left(f_{X^{2}}\right) \geq 2(K-1)>K$, a contradiction.

Let $\alpha(K)$ denote the worst payoff for player $i$ from a collective strategy in the set $T$ for the $K$-unit allocation problem,

$$
\alpha(K)=\inf \left\{V_{i}(f) \mid f \in T\right\}
$$

This expression does not depend on $i$ by symmetry of the game. We argue next that the infimum is a minimum.

Lemma 11.3: For every $i \in N$, there is a collective strategy $f \in T$ such that $V_{i}(f)=\alpha(K)$.

Proof: Suppose, there is no collective strategy $f \in T$ such that $V_{i}(f)=\alpha(K)$. Then there are non-empty subsets $X^{1}, X^{2}$ of $X$ such that $X^{1} \subset X^{2}$ and a sequence of natural numbers $\left\{m_{n}\right\}_{n \in \mathbb{N}}$ such that $f_{X^{1}, m_{n}, X^{2}} \in F_{*}$ and $V_{i}\left(f_{X^{1}, m_{n}, X^{2}}\right) \rightarrow \alpha(K)$ as $n \rightarrow \infty$. Since by Lemma 9.4 the sequence $V_{i}\left(f_{X^{1}, m_{n}, X^{2}}\right)$ converges to $V_{i}\left(f_{X^{1}}\right)$, we have $V_{i}\left(f_{X^{1}}\right)=\alpha(K)$. However, since $f_{X^{1}, m_{n}, X^{2}}$ is an element of $F_{*}$, so is $f_{X^{1}}$, yielding a contradiction.

The stationary collective equilibrium of Theorem 11.1 gives all players a payoff of $K / 3$, so $\alpha(K) \leq K / 3$. Theorem 11.4 shows that any collective equilibrium induced by two-step game-plans, i.e. any collective equilibrium in $T$, leads to approximately the same payoffs when $K$ is sufficiently large.

THEOREM 11.4: It holds that

$$
\lim _{K \rightarrow \infty} \frac{\alpha(K)}{K}=\frac{1}{3}
$$


Proof: Fix some $K$ and define $\alpha=\alpha(K)$. If $y \in Y_{*}$ then there is $f \in F_{*}$ such that $y \in \mathrm{WD}(f)$. Hence there are at least two players $i$ such that $y_{i} \geq V_{i}(f) \geq \alpha$. We therefore have the inclusion

$$
Y_{*} \subset\left\{x \in X||\left\{i \in N: x_{i} \geq \alpha\right\} \mid \geq 2\right\} .
$$

We will put a lower bound on the equilibrium payoff of, without loss of generality, Player 3. To do so, for given continuation utilities $z \in \mathbb{R}_{+}^{3}$, we compute the expected payoffs $\phi(z)$ to Player 3 resulting from a collective strategy that rejects an alternative $x$ if and only if $\left(x_{1}, x_{3}\right) \ll(\alpha, \alpha)$ or $\left(x_{2}, x_{3}\right) \ll(\alpha, \alpha)$, or $\left(x_{1}, x_{2}\right) \leq\left(z_{1}, z_{2}\right)$, pretending that the continuation utilities are indeed equal to $z$. More precisely, we have

$$
\phi(z)=\frac{1}{|X|} \sum_{x \in X} \phi_{x}(z)
$$

where

$$
\phi_{x}(z)=\left\{\begin{array}{l}
z_{3}, \text { if }\left[x_{1}<\alpha \text { and } x_{3}<\alpha\right] \text { or }\left[x_{2}<\alpha \text { and } x_{3}<\alpha\right] \text { or }\left[x_{1} \leq z_{1} \text { and } x_{2} \leq z_{2}\right] \\
x_{3}, \text { otherwise. }
\end{array}\right.
$$

We consider continuation utilities in the set $Z$ defined by

$$
Z=\left\{z \in \mathbb{R}_{+}^{3} \mid z_{1}+z_{2}+z_{3}=K, z_{1} \geq \alpha, z_{2} \geq \alpha, z_{3}=\alpha\right\}
$$

The next lemma shows that the evaluation of $\phi$ on $Z$ provides a lower bound for $\alpha$.

LEMma 11.5: There exists $\bar{z} \in Z$ such that $\phi(\bar{z}) \leq \alpha$.

ProOF: Let $f_{X^{1}, m, X^{2}} \in F_{*}$ be such that $\alpha=V_{3}\left(f_{X^{1}, m, X^{2}}\right)$ and let $z=V\left(f_{X^{1}, m-1, X^{2}}\right)$. If $m=0$ we set $X^{1}=X^{2}$ and have $f_{X^{1}, m-1, X^{2}}$ denote the collective strategy $f_{X^{2}}$. We observe that $z_{1}+z_{2}+z_{3}=K$, and $z \geq(\alpha, \alpha, \alpha)$ since $f_{X^{1}, m-1, X^{2}}$ belongs to $F_{*}$.

Consider some $x \in X^{1}$. We have $x_{i} \geq \alpha$ for at least two players $i$, since $X^{1} \subset Y_{*}$. If $\phi_{x}(z) \neq x_{3}$, then $\phi_{x}(z)=z_{3}, x_{1} \leq z_{1}$ and $x_{2} \leq z_{2}$, which implies $z_{3} \leq x_{3}$. Otherwise, it holds that $\phi_{x}(z)=x_{3}$. In both cases we have $\phi_{x}(z) \leq x_{3}$.

Consider some $x \in X \backslash X^{1}$. Since $f_{X^{1}, m, X^{2}}$ is an element of $F_{*}$ it holds that $\operatorname{SD}\left(f_{X^{1}, m-1, X^{2}}\right) \subset$ $X^{1}$, so there is at most one player $i$ such that $x_{i}>z_{i}$. If $\phi_{x}(z) \neq z_{3}$, then $\phi_{x}(z)=x_{3}$, and $x_{1}>z_{1}$ or $x_{2}>z_{2}$. It follows that $x_{3} \leq z_{3}$. Otherwise, it holds that $\phi_{x}(z)=z_{3}$. In both cases we have $\phi_{x}(z) \leq z_{3}$.

It holds that

$$
\phi(z)=\frac{1}{|X|} \sum_{x \in X} \phi_{x}(z) \leq \frac{1}{|X|}\left(\sum_{x \in X^{1}} x_{3}+\sum_{x \in X \backslash X^{1}} z_{3}\right)=V_{3}\left(f_{X^{1}, m, X^{2}}\right)=\alpha .
$$


We define $\bar{z}=\left(z_{1}+z_{3}-\alpha, z_{2}, \alpha\right)$. We show that, for each $x \in X, \phi_{x}(\bar{z}) \leq \phi_{x}(z)$, which implies $\phi(\bar{z}) \leq \phi(z)$ and proves the lemma. If $\left[x_{1}<\alpha\right.$ and $\left.x_{3}<\alpha\right]$ or $\left[x_{2}<\alpha\right.$ and $\left.x_{3}<\alpha\right]$ or $\left[x_{1} \leq z_{1}\right.$ and $\left.x_{2} \leq z_{2}\right]$, then $\phi_{x}(z)=z_{3}$ and $\phi_{x}(\bar{z})=\bar{z}_{3}$, and the result follows since $\bar{z}_{3}=\alpha \leq z_{3}$. Otherwise, we have $\phi_{x}(z)=x_{3}$. If $\phi_{x}(\bar{z})=\bar{z}_{3}$ we must have $x_{1} \leq \bar{z}_{1}$ and $x_{2} \leq \bar{z}_{2}$, so $\bar{z}_{3} \leq x_{3}$ as desired.

We continue by deriving a lower bound on $\phi(\bar{z})$ for $\bar{z} \in Z$, which in turn serves as the lower bound on $\alpha$ presented in the next lemma.

LEMMA 11.6: It holds that

$$
\alpha \geq \frac{1}{3} K+\frac{8 \alpha^{3}-(K-\alpha+2)^{3}}{4(K+1)(K+2)} .
$$

Proof: Take some $\bar{z} \in Z$ satisfying the conditions of Lemma 11.5. We show that the right-hand side of the inequality in Lemma 11.6 is a lower bound for $\phi(\bar{z})$.

For $r \in \mathbb{R}$, let $\lfloor r\rfloor$ be the greatest integer less than or equal to $r$, and $\lceil r\rceil$ be the smallest integer greater than or equal to $r$. We define the subsets $C^{1}, C^{2}$, and $C^{3}$ of $X$ by

$$
\begin{aligned}
& C^{1}=\left\{x \in X \mid x_{1}<\alpha, x_{3}<\alpha\right\} \quad=\left\{x \in X \mid x_{1} \leq\lceil\alpha\rceil-1, x_{3} \leq\lceil\alpha\rceil-1\right\}, \\
& C^{2}=\left\{x \in X \mid x_{2}<\alpha, x_{3}<\alpha\right\}=\left\{x \in X \mid x_{2} \leq\lceil\alpha\rceil-1, x_{3} \leq\lceil\alpha\rceil-1\right\} \\
& C^{3}=\left\{x \in X \mid x_{1} \leq \bar{z}_{1}, x_{2} \leq \bar{z}_{2}\right\}=\left\{x \in X \mid x_{1} \leq\left\lfloor\bar{z}_{1}\right\rfloor, x_{2} \leq\left\lfloor\bar{z}_{2}\right\rfloor\right\}
\end{aligned}
$$

and define $C=C^{1} \cup C^{2} \cup C^{3}$. Since

$$
\begin{aligned}
V_{3}\left(f_{X}\right) & =\frac{|X \backslash C|}{|X|} V_{3}\left(f_{X \backslash C}\right)+\frac{|C|}{|X|} V_{3}\left(f_{C}\right)=\frac{1}{3} K, \\
V\left(f_{C}\right) & =\sum_{i=1,2,3} \frac{\left|C^{i}\right|}{|C|} V\left(f_{C^{i}}\right),
\end{aligned}
$$

it holds that

$$
\begin{aligned}
\phi(\bar{z}) & =\frac{|C|}{|X|} \bar{z}_{3}+\frac{|X \backslash C|}{|X|} V_{3}\left(f_{X \backslash C}\right)=\frac{|C|}{|X|} \bar{z}_{3}+\frac{1}{3} K-\frac{|C|}{|X|} V_{3}\left(f_{C}\right) \\
& =\frac{1}{3} K+\sum_{i=1,2,3} \frac{\left|C^{i}\right|}{|X|}\left(\bar{z}_{3}-V_{3}\left(f_{C^{i}}\right)\right) .
\end{aligned}
$$

A simple calculation reveals that

$$
\begin{aligned}
\left|C^{1}\right| & =\lceil\alpha\rceil^{2}, & & V_{3}\left(f_{C^{1}}\right)=\frac{1}{2}(\lceil\alpha\rceil-1), \\
\left|C^{2}\right| & =\lceil\alpha\rceil^{2}, & & V_{3}\left(f_{C^{2}}\right)=\frac{1}{2}(\lceil\alpha\rceil-1), \\
\left|C^{3}\right| & =\left(\left\lfloor\bar{z}_{1}\right\rfloor+1\right)\left(\left\lfloor\bar{z}_{2}\right\rfloor+1\right), & V_{3}\left(f_{C^{3}}\right) & =K-\frac{1}{2}\left\lfloor\bar{z}_{1}\right\rfloor-\frac{1}{2}\left\lfloor\bar{z}_{2}\right\rfloor,
\end{aligned}
$$


SO

$$
\begin{aligned}
& \bar{z}_{3}-V_{3}\left(f_{C^{1}}\right)=\alpha-\frac{1}{2}(\lceil\alpha\rceil-1) \geq \frac{1}{2} \alpha \\
& \bar{z}_{3}-V_{3}\left(f_{C^{2}}\right)=\alpha-\frac{1}{2}(\lceil\alpha\rceil-1) \geq \frac{1}{2} \alpha \\
& \bar{z}_{3}-V_{3}\left(f_{C^{3}}\right)=\bar{z}_{3}-K+\frac{1}{2}\left\lfloor\bar{z}_{1}\right\rfloor+\frac{1}{2}\left\lfloor\bar{z}_{2}\right\rfloor \geq \alpha-K+\frac{1}{2} \bar{z}_{1}+\frac{1}{2} \bar{z}_{2}-1=-\frac{1}{2}(K-\alpha+2) .
\end{aligned}
$$

It is immediate that

$$
\begin{aligned}
& \left|C^{1}\right|\left(\bar{z}_{3}-V_{3}\left(f_{C^{1}}\right)\right) \geq\lceil\alpha\rceil^{2} \frac{1}{2} \alpha \geq \frac{1}{2} \alpha^{3}, \\
& \left|C^{2}\right|\left(\bar{z}_{3}-V_{3}\left(f_{C^{2}}\right)\right) \geq\lceil\alpha\rceil^{2} \frac{1}{2} \alpha \geq \frac{1}{2} \alpha^{3} .
\end{aligned}
$$

Using the fact that $a b \leq(a+b)^{2} / 4$, we derive

$$
\left|C^{3}\right| \leq \frac{1}{4}\left(\left\lfloor\bar{z}_{1}\right\rfloor+1+\left\lfloor\bar{z}_{2}\right\rfloor+1\right)^{2} \leq \frac{1}{4}\left(\bar{z}_{1}+\bar{z}_{2}+2\right)^{2}=\frac{1}{4}(K-\alpha+2)^{2} .
$$

Therefore it holds that

$$
\left|C^{3}\right|\left(\bar{z}_{3}-V_{3}\left(f_{C^{3}}\right)\right) \geq-\frac{1}{8}(K-\alpha+2)^{3} .
$$

Finally, we observe that

$$
|X|=\sum_{k=0}^{K}(K+1-k)=\frac{1}{2}(K+1)(K+2) .
$$

After substituting (11.3)-(11.6) into (11.2), we find the desired lower bound.

To complete the proof of the theorem, assume without loss of generality that the sequence $\alpha(K) / K$ converges to some non-negative limit $\beta$. From Lemma 11.6 we obtain

$$
\beta=\lim _{K \rightarrow \infty} \frac{\alpha(K)}{K} \geq \frac{1}{3}+\lim _{K \rightarrow \infty} \frac{8 \alpha^{3}-(K-\alpha+2)^{3}}{4 K(K+1)(K+2)}=\frac{1}{3}+2 \beta^{3}-\frac{1}{4}(1-\beta)^{3} .
$$

After some rewriting, we find this expression to be equivalent to

$$
(1-3 \beta)^{2}(1+3 \beta) \leq 0
$$

The only non-negative value for $\beta$ satisfying the latter inequality is $\beta=1 / 3$. 


\section{Robustness of collective equilibria}

Examples 4.4 and 4.5 show that collective stopping games may not have stationary equilibria. In the presence of breakdown, stationary equilibria are guaranteed to exist, but may be in mixed strategies as demonstrated by Example 4.6. It can be hard to compute the exact probabilities in a mixed strategy that are needed to make the players indifferent between accepting and rejecting a particular alternative, and, moreover, these probabilities change when the utilities of the alternatives or the transition probabilities in the selection dynamics change. We argue next that collective equilibria generated by two-step gameplans on the other hand are robust to small changes in utilities and in Markov transition probabilities that govern the selection of alternatives.

To do so, we fix the set of players, alternatives, and the decision rules used, and parameterize a game $\Gamma(\mu, u)$ by the Markov process $\mu$ that governs the choice of alternatives and the profile of utility functions $u$. Since the probability distribution $\mu^{0}$ from which alternatives are initially drawn only influences equilibrium utilities, but not the accept/reject decisions themselves, we do not use it in our parametrization. We identify $\mu$ with its stochastic matrix of transition probabilities. The set of stochastic matrices is denoted by $\mathcal{M}$. The profile of utility functions $u$ is identified with a point in $\mathcal{U}=\mathbb{R}_{+}^{N \times X}$.

A key property of strategies induced by two-step game-plans is the following.

Lemma 12.1: If $f$ is induced by two-step game-plans, then $\{f[s] \mid s \in S\}$ is a finite set.

Proof: If $f$ is a two-step collective strategy, then the cardinality of $\{f[s] \mid s \in S\}$ is at most $m+1$, where $m$ is the threshold of $f$.

Next consider the case where $f$ is induced by a tuple $\mathcal{F}=\left(f_{0}, f_{x}: x \in X\right)$ of two-step strategies. For each $s \in S$, the collective strategy $f[s]$ is induced by the tuple $\left(g, f_{x}: x \in X\right)$ where $g$ equals $h[r]$ for some member $h$ of $\mathcal{F}$ and $r \in\{\emptyset\} \cup S$. By the observation in the previous paragraph there are at most finitely many of such strategies $g$, and the result follows.

We let $V_{x, i}^{\mu, u}(f)$ denote the payoff to player $i$ in the game $\Gamma_{x}(\mu, u)$ under the collective strategy $f$.

Lemma 12.2: Let $f$ be a collective strategy induced by two-step game-plans. Then the function $V_{x, i}^{\mu, u}(f)$ is continuous at each point of $\mathcal{M} \times \mathcal{U}$.

Proof: We first prove the lemma for stationary strategies. Let $f=f_{X^{1}}$ where $X^{1}$ is a non-empty subset of $X$. In view of Equation (9.2) it is sufficient to prove that $V_{x, i}^{\mu, u}\left(f_{X^{1}}\right)$ 
is a continuous function of $(\mu, u)$ for each $x \in X \backslash X^{1}$. Letting

$$
v_{y}=V_{y, i}^{\mu, u}\left(f_{X^{1}}\right), a_{y}=\sum_{z \in X^{1}} \mu(z \mid y) u_{i}(z), \text { and } A_{x y}=\mu(y \mid x)
$$

for all $x$ and $y$ in $X \backslash X^{1}$ we can rewrite (9.2) in the vector-matrix notation as $v=a+A v$. As we have argued in the proof of Lemma 9.4, the spectral radius of $A$ is smaller than one. This means that the matrix $(I-A)$ is invertible. Hence $v=(I-A)^{-1} a$, an expression that is continuous in $A$ and $a$. Clearly $A$ and $a$ are continuous as functions of $(\mu, u)$ on $\mathcal{M} \times \mathcal{U}$.

Equation (9.1) implies that if $V_{x, i}\left(f_{X^{1}, m, X^{2}}\right)$ is continuous in $(\mu, u)$ for each $x \in X$, so is $V_{x, i}\left(f_{X^{1}, m+1, X^{2}}\right)$ for each $x \in X$. This establishes the lemma for any two-step collective strategy.

Finally consider a collective strategy $f$ induced by two-step game-plans. Let $f_{0}$ be the collective strategy defining the main game-plan of $f$, so $V_{x, i}^{\mu, u}(f)=V_{x, i}^{\mu, u}\left(f_{0}\right)$. Since $f_{0}$ is two-step, the result follows from the conclusion of the preceding paragraph.

Consider a collective equilibrium $f \in T$ of the game $\Gamma(\bar{\mu}, \bar{u})$. It is said to be robust if it is a collective equilibrium of the game $\Gamma(\mu, u)$ for each $(\mu, u)$ in some open neighborhood of $(\bar{\mu}, \bar{u})$. A subset of $\mathcal{U}$ is said to be negligible if it is meager and has Lebesgue measure zero.

THEOREM 12.3: There is a set $\mathcal{G} \subset \mathcal{M} \times \mathcal{U}$ such that

[1] For each $(\mu, u) \in \mathcal{G}$, every collective equilibrium $f \in T$ is robust.

[2] For each $\mu \in \mathcal{M}$, the complement of the $\mu$-section of the set $\mathcal{G}$ in $\mathcal{U}$ is negligible as a subset of $\mathcal{U}$.

Proof: It will be convenient to write the payoff on $f$ as

$$
V_{x, i}^{\mu, u}(f)=\sum_{y \in X} \nu^{\mu}(f, x ; y) u_{i}(y)
$$

where $\nu^{\mu}(f, x ; y)$ is the probability that the play of the game $\Gamma_{x}(\mu, u)$ terminates with the acceptance of the alternative $y$. Let

$$
T^{\mu}=\bigcup_{x \in X}\left\{f \in T \mid \nu^{\mu}(f, x ; x)=1\right\} .
$$

If $f \in T^{\mu}$ then there is an $x \in X$ such that $V_{x, i}^{\mu, u}(f)=u_{i}(x)$ for all $u \in \mathcal{U}$ and all $i \in N$. For each $f \in T$ define the subset $\mathcal{U}_{f}^{\mu}$ of $\mathcal{U}$ as follows: If $f$ is an element of $T^{\mu}$ we set

$$
\mathcal{U}_{f}^{\mu}=\left\{u \in \mathcal{U}: u_{i}(x) \neq u_{i}(y) \text { for all }(i, x, y) \in N \times X \times X \text { with } x \neq y\right\} .
$$


If $f$ is not an element of $T^{\mu}$ we set

$$
\mathcal{U}_{f}^{\mu}=\left\{u \in \mathcal{U}: u_{i}(x) \neq V_{x, i}^{\mu, u}(f) \text { for all }(i, x) \in N \times X\right\}
$$

We define

$$
\mathcal{U}^{\mu}=\bigcap_{f \in T} \mathcal{U}_{f}^{\mu} \text { and } \mathcal{G}=\bigcup_{\mu \in \mathcal{M}}\{\mu\} \times \mathcal{U}^{\mu}
$$

We complete the proof by showing that the set $\mathcal{G}$ thus defined has properties [1] and [2] required by the theorem.

Clearly $\mathcal{U}^{\mu}$ is a $\mu$-section of $\mathcal{G}$. We show that the complement of $\mathcal{U}^{\mu}$ is negligible. Since the set $T$ is countable, it suffices to show that for each $f \in T$ the complement of $\mathcal{U}_{f}^{\mu}$ is negligible.

First consider the case $f \in T^{\mu}$. In this case the complement of $\mathcal{U}_{f}^{\mu}$ is covered by finitely many sets of the form $\left\{u \in \mathcal{U} \mid u_{i}(x)=u_{i}(y)\right\}$ where $i \in N$ and $x \neq y$. Each such set is a lower-dimensional subspace of $\mathcal{U}$ and hence a negligible set.

Suppose $f$ is not in $T^{\mu}$. The complement of $\mathcal{U}_{f}^{\mu}$ is then covered by finitely many sets of the form $C=\left\{u \in \mathcal{U} \mid u_{i}(x)=V_{x, i}^{\mu, u}(f)\right\}$. Since the payoff $V_{x, i}^{\mu, u}(f)$ is linear in $u$, the set $C$ is a linear subspace of $\mathcal{U}$. It is a proper subset of $\mathcal{U}$ because $\nu^{\mu}(f, x ; x)<1$. We conclude that $C$ is a lower-dimensional subspace of $\mathcal{U}$ and is hence negligible.

Take a game $(\bar{\mu}, \bar{u}) \in \mathcal{G}$. For each $f \in T$, we define an open neighborhood of $(\bar{\mu}, \bar{u})$ by letting

$$
\mathcal{O}(f)=\bigcap_{i \in N} \bigcap_{x \in X}\left\{(\mu, u) \in \mathcal{M} \times \mathcal{U} \mid \begin{array}{l}
\bar{u}_{i}(x)>V_{x, i}^{\bar{\mu}, \bar{u}}(f) \text { implies } u_{i}(x)>V_{x, i}^{\mu, u}(f) \\
\bar{u}_{i}(x)<V_{x, i}^{\bar{\mu}, \bar{u}}(f) \text { implies } u_{i}(x)<V_{x, i}^{\mu, u}(f)
\end{array}\right\} .
$$

Invoking the definition of $\mathcal{U}^{\bar{\mu}}$ it is easy to see that for every game $(\mu, u) \in \mathcal{O}(f)$ the alternative $x \in X$ strictly (weakly) dominates $f$ in the game $\Gamma(\mu, u)$ if and only if it does so in the game $\Gamma(\bar{\mu}, \bar{u})$.

Let $\bar{f} \in T$ be a collective equilibrium of $\Gamma(\bar{\mu}, \bar{u})$. We argue that $\bar{f}$ is robust. We define

$$
\mathcal{O}=\bigcap_{s \in S} \mathcal{O}(\bar{f}[s])
$$

the intersection being finite by Lemma 12.1. Take any $(\mu, u) \in \mathcal{O}$. By the remark in the previous paragraph, for every $s \in S$, the alternative $x$ strictly (weakly) dominates $\bar{f}[s]$ in the game $\Gamma(\mu, u)$ if and only if it does so in the game $\Gamma(\bar{\mu}, \bar{u})$. It follows directly from Definition 6.2 that $\bar{f}$ is a collective equilibrium of $\Gamma(\mu, u)$, as desired. 


\section{Incomplete information}

In this section, we generalize our model by allowing the voting order to be probabilistic and history-dependent, and information to be incomplete. We continue to assume that players observe the moves by nature, but we do no longer require that a player observes the actions of other players.

We therefore consider a game $\Gamma^{\prime}$ defined as follows. In each period $t=0,1, \ldots$ nature draws an alternative $x$ from a set $X$ according to the Markov process $\mu$ on $X$, where the selection in period 0 occurs according to the probability distribution $\mu^{0}$ on $X$. Then nature selects the first player to vote on $x$, say player $i_{1} \in N$. After Player $i_{1}$ casts a "y" or an "n" vote, nature chooses the next player to cast a vote, say player $i_{2} \in N \backslash\left\{i_{1}\right\}$, who casts a "y" or an "n" vote. Next nature chooses player $i_{3} \in N \backslash\left\{i_{1}, i_{2}\right\}$, and so on until all players have voted. The choice of a responder $i_{k}$ may be probabilistic and may depend on the entire history of play. If the set of players who voted " $\mathrm{y}$ " is decisive, the game ends and player $i \in N$ receives utility $u_{i}(x)$. Otherwise the next period begins. Perpetual disagreement results in utility zero for all players.

Let $H_{i}$ denote the set of finite histories after which it is player $i$ 's turn to cast a vote. The description of the game $\Gamma^{\prime}$ is completed by specifying the information sets for every player: $\mathcal{H}_{i}$ denotes the information partition of $H_{i}$ for player $i$. Given a history $h \in H_{i}, t_{h}$ denotes the current period at $h$, and corresponds to the number of alternatives that have been voted down so far. For $t=0, \ldots, t_{h}$, the alternative chosen by nature in period $t$ is denoted by $x_{t}(h)$ and for $t=0, \ldots, t_{h}-1$, we write $a_{t}^{i}(h)$ for the action taken by player $i$ in period $t$. With respect to the information structure of the game we make the following assumption.

[A5] If $h, h^{\prime}$ belong to the same information set $I_{i}$ in $\mathcal{H}_{i}$, then

1. $t_{h}=t_{h^{\prime}}$,

2. for every $t=0, \ldots, t_{h}, x_{t}(h)=x_{t}\left(h^{\prime}\right)$,

3. for every $t=0, \ldots, t_{h}-1, a_{t}^{i}(h)=a_{t}^{i}\left(h^{\prime}\right)$.

Since we allow for incomplete information, besides the case with sequential voting, we now also cover cases where some or all of the players cast their votes simultaneously. For example, we allow for the case where all players vote simultaneously and the votes are never revealed, as well as the case where all players vote simultaneously and all votes cast in a given period are revealed at the the end of that period. 
A strategy of player $i$ is a map $\sigma_{i}: \mathcal{H}_{i} \rightarrow[0,1]$. A sequential equilibrium of $\Gamma^{\prime}$ specifies a joint strategy as well as the beliefs that each player has regarding the relative likelihood of different histories in the same information set. A belief system of player $i$ specifies a probability distributions $\beta_{i}\left(I_{i}\right)$ on the set $I_{i}$ for each $I_{i} \in \mathcal{H}_{i}$. A sequential equilibrium is defined as a pair $(\sigma, \beta)$ such that $\sigma$ is a joint strategy and $\beta$ a belief system such that $\sigma$ is sequentially rational given $\beta$, and $\beta$ is consistent with $\sigma$.

A joint strategy $\sigma$ is said to be sequentially rational given the belief system $\beta$ if for every player $i \in N$ and each of player $i$ 's information sets $I_{i} \in \mathcal{H}_{i}$, the strategy $\sigma_{i}$ is a best response against the joint strategy $\sigma$ conditional on reaching information set $I_{i}$ given the belief system $\beta_{i}\left(I_{i}\right)$. The condition of consistency encompasses the requirement that $\beta$ be derived from $\sigma$ using Bayes' rule and it also imposes some restrictions on how $\beta_{i}\left(I_{i}\right)$ should be defined for those information sets $I_{i}$ that are reached with probability zero. We deliberately avoid stating a more precise definition of consistency since the result below is valid for any definition of consistency satisfying the extremely mild requirement that for every joint strategy $\sigma$ there is at least one system of beliefs consistent with $\sigma$. Hence we simply assume that some definition of consistency satisfying this requirement is used and we write $L(\sigma)$ to denote the set of beliefs consistent with the joint strategy $\sigma$.

Notice that each action-independent strategy $\sigma_{i}$ of the game $\Gamma$ naturally induces a strategy $\sigma_{i}^{\prime}$ in the game $\Gamma^{\prime}$. For $I_{i} \in \mathcal{H}_{i}$, choose $h \in I_{i}$, define $s \in S$ by $s_{t}=x_{t}(h)$ for $t=0, \ldots, t_{h}$, and let $\sigma_{i}^{\prime}\left(I_{i}\right)$ be equal to $\sigma_{i}(s)$. We can now state the following result:

THEOREM 13.1: Let $\sigma$ be a subgame perfect equilibrium of $\Gamma$ in pure actionindependent strategies. Then $\left(\sigma^{\prime}, \beta\right)$ is a sequential equilibrium of $\Gamma^{\prime}$ whenever $\beta \in L\left(\sigma^{\prime}\right)$.

The intuition for the result is straightforward: If all players use an action-independent strategy, then any additional information contained in the information partition $\mathcal{H}_{i}$ is irrelevant: a strategy is a sequentially rational (or not) irrespective of the beliefs. We spell out the proof at the risk of proving the obvious. Consider an information set $I_{i} \in \mathcal{H}_{i}$, $h \in I_{i}$, and define $s \in S$ by $s_{t}=x_{t}(h)$ for $t=0, \ldots, t_{h}$. Since $\sigma$ is a subgame perfect equilibrium of $\Gamma$, the strategy $\sigma_{i}$ is a best response for player $i$ in each subgame of $\Gamma$ that follows the rejection of the alternatives $s_{0}, \ldots, s_{t}$. It follows that $\sigma_{i}$ is a best response in $\Gamma^{\prime}$ conditional on reaching $I_{i}$ when player $i$ puts probability 1 on any given information node $h \in I_{i}$. It then follows that $\sigma_{i}$ is a best response under any probability distribution that player $i$ might have on $I_{i}$. 


\section{Conclusion}

Collective stopping games form a rich class of games that include collective choice games, quitting games, and coalition formation games as special cases. Particular examples of collective stopping games involve recruitment decisions by committees, business financing decisions, and oligopoly exit decisions. The standard equilibrium concept to analyze such games, stationary equilibrium in subgame perfect strategies, is problematic as for collective stopping games it may not exist at all or only exist in mixed strategies. Moreover, the existing literature has only demonstrated the existence of subgame perfect $\epsilon$-equilibria. This triggers the question whether even subgame perfect equilibria may not always exist for such games.

In this paper we show not only that subgame perfect equilibria always exist in collective stopping games, but also that we can define a finite set of strategies which is guaranteed to contain a subgame perfect equilibrium. We require strategies to be pure and action-independent, where the latter property has the advantage that we can incorporate various degrees of incomplete information as far as the voting behavior of the players is concerned. Action-independence also allows us to summarize the play of the game by collective strategies. We require collective strategies to be simple and to be generated by two-step game-plans. Under a two-step game-plan, the game ends when as soon as nature draws an alternative from some set $X_{1}$ in the first $m$ periods, or when nature draws an alternative from a bigger set $X_{2}$ in a later period. Since we can formulate an upper bound on $m$, this leads to the desired finite set of strategies which is shown to contain a subgame perfect equilibrium.

A particular example is the case of three alternatives, where utilities of the players and decision rules for the acceptance of an alternative are such that a Condorcet cycle results. Such examples can easily occur in the context of collective choice or in coalition formation processes. In such an example stationary equilibria may not exist at all. Subgame perfect equilibria in pure and action-independent strategies do exist, where the induced collective strategy is such that any alternative is accepted in period 0 and the unlawful rejection of an alternative is followed by a particular two-step punishment game-plan. In the BaronFerejohn model of redistributive politics, stationary equilibria do exist. We show that when

the units that can be used for redistributive purposes are sufficiently small, the payoffs of collective equilibria induced by two-step game-plans are arbitrarily close to the payoffs from a stationary equilibrium. 


\section{References}

Abreu, D. (1988), "On the Theory of Infinitely Repeated Games with Discounting," Econometrica, 56, 383-396.

Banks, J., And J. Duggan (2000), "A Bargaining Model of Collective Choice," American Political Science Review, 94, 73-88.

Baron, D.P., And J.A. Ferejohn (1989), "Bargaining in Legislatures," American Political Science Review, 83, 1181-1206.

Bernheim, B. D., And S. N. Slavov (2009), "A Solution Concept for Majority Rule in Dynamic Settings," Review of Economic Studies, 76, 33-62.

Blackwell, D., And T. Ferguson (1968), "The Big Match," Annals of Mathematical Statistics, 39, 159-163.

BlOCH, F. (1996), "Sequential Formation of Coalitions in Games with Externalities and Fixed Payoff Division," Games and Economic Behavior, 14, 90-123.

Bloch, F., And E. Diamantoudi (2011), "Noncooperative Formation of Coalitions in Hedonic Games," International Journal of Game Theory, 40, 263-280.

Breton, M. Le (1987), "On the Core of Voting Games," Social Choice and Welfare, 4, 295-305.

Compte, O., And P. Jehiel (2010A), "Bargaining and Majority Rules: A Collective Search Perspective," Journal of Political Economy, 118, 189-221.

Compte, O., And P. Jehiel (2010B), "The Coalitional Nash Bargaining Solution," Econometrica, 78, 1593-1623.

Cox, G.W. (1984), "Non-Collegial Simple Games and the Nowhere Denseness of the Set of Preference Profiles Having a Core," Social Choice and Welfare, 1, 159-164.

Duggan, J. (2011), "Coalitional Bargaining Equilibria," available at http://johnduggan.net/.

Fink, A.M. (1964), "Equilibrium in a Stochastic n-Person Game," Journal of Science of Hiroshima University Series A-I, 28, 89-93.

Flesch, J., J. Kuipers, G. Schoenmakers, And K. Vrieze (2010), "Subgame Perfection in Positive Recursive Games with Perfect Information," Mathematics of Operations Research, 35, 193-207.

Fudenberg, D., And J. Tirole (1991), Game Theory, MiT Press, Cambridge, Massachusetts.

Herings, P.J.J., AND H. Houba (2010), "The Condorcet Paradox Revisited," METEOR Research Memorandum 10/09, Maastricht University, Maastricht, pp. 1-45.

Horn, R.A., And C.R. Johnson (2007), Matrix Analysis, Cambridge University Press, Cambridge.

Konishi, H., And D. RaY (2003), "Coalition Formation as a Dynamic Process," Journal of Economic Theory, 110, 1-41.

Mashiah-YaAkovi, A. (2009), "Periodic Stopping Games," International Journal of Game Theory, 38, $169-181$. 
Maskin, E.S., And J. Tirole (2001), "Markov Perfect Equilibrium, I. Observable Actions," Journal of Economic Theory, 100, 191-219.

Maynard-Smith, J. (1974), "The Theory of Games and the Evolution of Animal Conflicts," Journal of Theoretical Biology, 47, 209-221.

OkadA, A. (1996), "A Noncooperative Coalitional Bargaining Game with Random Proposers," Games and Economic Behavior, 16, 97-108.

Peleg, B., And H. Peters (2010), Strategic Social Choice, Stable Representations of Constitutions, Springer-Verlag, Berlin.

Penn, E.M. (2009), "A Model of Farsighted Voting," American Journal of Political Science, 53, 36-54.

Plott, C.R. (1967), "A Notion of Equilibrium and its Possibility Under Majority Rule," American Economic Review, 57, 787-806.

Ray, D., And R. Vohra (1999), "A Theory of Endogenous Coalition Structures," Games and Economic Behavior, 26, 286-336.

Roberts, K. (2007), "Condorcet Cycles? A Model of Intertemporal Voting," Social Choice and Welfare, 29, 383-404.

Rubinstein, A. (1979), "A Note about the "Nowhere Denseness" of Societies Having an Equilibrium under Majority Rule," Econometrica, 47, 511-514.

Schofield, N. (1983), "Generic Instability of Majority Rule," Review of Economic Studies, 50, 695705.

Sobel, M.J. (1971), "Noncooperative Stochastic Games," The Annals of Mathematical Studies, 42, $1930-1935$.

Solan, E. (2005), "Subgame-perfection in Quitting Games with Perfect Information and Differential Equations," Mathematics of Operations Research, 30, 51-72.

Solan, E., AND N. Vieille (2001), "Quitting Games," Mathematics of Operations Research, 26, 265285.

Takahashi, M. (1964), "Equilibrium Points of Stochastic Non-cooperative $n$-Person Games," Journal of Science of Hiroshima University Series A-I, 28, 95-99.

Vartiainen, H. (2011), "Dynamic Coalitional Equilibrium," Journal of Economic Theory, 146, 672698. 